\title{
COMPLETENESS AND CATEGORICITY (IN POWER): FORMALIZATION WITHOUT FOUNDATIONALISM
}

Author(s): JOHN T. BALDWIN

Source: The Bulletin of Symbolic Logic, Vol. 20, No. 1 (MARCH 2014), pp. 39-79

Published by: Association for Symbolic Logic

Stable URL: http://www.jstor.org/stable/23800699

Accessed: 04-03-2015 16:59 UTC

Your use of the JSTOR archive indicates your acceptance of the Terms \& Conditions of Use, available at http://www.jstor.org/page/info/about/policies/terms.jsp

JSTOR is a not-for-profit service that helps scholars, researchers, and students discover, use, and build upon a wide range of content in a trusted digital archive. We use information technology and tools to increase productivity and facilitate new forms of scholarship. For more information about JSTOR, please contact support@jstor.org. 


\title{
COMPLETENESS AND CATEGORICITY (IN POWER): FORMALIZATION WITHOUT FOUNDATIONALISM*
}

\author{
JOHN T. BALDWIN
}

\begin{abstract}
We propose a criterion to regard a property of a theory (in first or second order logic) as virtuous: the property must have significant mathematical consequences for the theory (or its models). We then rehearse results of Ajtai. Marek. Magidor. H. Friedman and Solovay to argue that for second order logic. "categoricity' has little virtue. For first order logic. categoricity is trivial: but 'categoricity in power' has enormous structural consequences for any of the theories satisfying it. The stability hierarchy extends this virtue to other complete theories. The interaction of model theory and traditional mathematics is examined by considering the views of such as Bourbaki. Hrushovski. Kazhdan. and Shelah to flesh out the argument that the main impact of formal methods on mathematics is using formal definability to obtain results in 'mainstream' mathematics. Moreover. these methods (e.g.. the stability hierarchy) provide an organization for much mathematics which gives specific content to dreams of Bourbaki about the architecture of mathematics.
\end{abstract}

§1. What is the role of categoricity?. In correspondence in 2008, Michael Detlefsen raised a number of questions about the role of categoricity. We discuss two of them in this paper.

Question $I^{1}$ : (A) Which view is the more plausible-that theories are the better the more nearly they are categorical, or that theories are the better the more they give rise to significant nonisomorphic interpretations?

(B) Is there a single answer to the preceding question? Or is it rather the case that categoricity is a virtue in some theories but not in others? If so, how do we tell these apart, and how to we justify the claim that categoricity is or would be a virtue just in the former?

Question II: Given that categoricity can rarely be achieved, are there alternative conditions that are more widely achievable and that give at least a substantial part of the benefit that categoricity would? Can completeness be shown to be such a condition? If so, can we give a relatively precise statement and demonstration of the part of the value of categoricity that it preserves?

Received November 6. 2013.

Key words and phrases. Categoricity. formalization. model theory. foundationalism, completeness

${ }^{*}$ I realized while writing that the title was a subconscious homage to the splendid historical work on Completeness and Categoricity by Awodey and Reck [6].

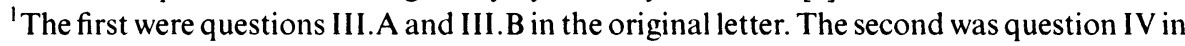
the Detlefsen letter. I thank Professor Detlefsen for permission to quote this correspondence. 
Further discussion revealed different understandings of some basic terminology. Does categoricity mean 'exactly one model', full stop? Or does it mean exactly one model in a given cardinal? Is a theory automatically closed under (deductive/semantic) consequence? Is the topic 'theory' or 'axiomatization'? Detlefsen's concerns were primarily about first or second order axiomatizations to provide descriptive completeness ${ }^{2}$ for a particular area or for all of mathematics. I was thinking mostly of arbitrary (complete) first order theories. These different perspectives led me to articulate two roles for formalism in mathematics: as a foundational tool and as one more device in the mathematician's toolbox. In developing this second role in Sections 4 and 5, I am also providing a counterpoint to earlier work of Kennedy [55] and myself [15] concerning formalism-free developments in logic.

After fixing precisely the meanings for this article of certain basic terminology, I begin the paper proper by analyzing Question I.B. What does it mean for a property of a theory to be virtuous? Before one can decide the virtue of categoricity, one must clarify what is meant by virtue. After providing a criterion for a 'good or virtuous property' in Section 2, we deal in the succeeding sections with Question I.A. Section 3 concerns 2 nd order logic, Section 4 first order logic and Section 5 infinitary logic. We argue in Section 3 that categoricity is interesting for a few second order sentences describing particular structures. But this interest arises from the importance of those axiomatizations of those structures; not from any intrinsic consequence of 2 nd order categoricity for arbitrary theories. From this standpoint, categoricity of a particular axiom set is crucial. In contrast, contemporary model theory focuses either on the properties of (complete) first order theories and classes of those theories or on studying particular theories, usually those arising in mathematical practice. Here, the particular choice of axioms is not important.

We answer question II by analyzing the importance of completeness. This analysis, coupled with our criteria for virtue (a virtuous property has significant mathematical consequences for theories or their models), leads to the main argument (Section 4) of this paper. Formalization impacts mathematical

\footnotetext{
${ }^{2}$ In [31], Detlefsen distinguishes between 'descriptive completeness' and 'completeness for truth'. Descriptive completeness connects a notion of a data set, containing say, a not fully determinate list of (perhaps formalized) propositions about some area (perhaps all) of mathematics and a set of formal propositions derived in a theory $T$. The theory $T$ is descriptively complete if the formal propositions contain all the formalized sentences expressing the data set. Gödel fixes on completeness for truth: every sentence in the formalized language is either derived or refuted by $T$. In this paper I discuss formalized theories. For them it seems evident that Gödel completeness is a sufficient but not necessarily necessary condition for descriptive completeness. (This is evident only on the assumption that the theory is in fact formalizing the data set in question. In a work in progress I analyze the foundations of geometry to examine in which logic (first order, $L_{\left(\omega_{1} . \omega\right.}$, second order one should formalize to be descriptive complete for 'Greek geometry'.) In general, when I speak of 'foundationalism', I refer to the goal of obtaining a basis for certitude in an area (perhaps) all of mathematics. The discussion of formalism as a tool for organizing mathematics argues that comparing various formalizations of local areas of mathematics allows one to see important patterns across mathematics without attempting any global foundations.
} 
practice most directly not because of its foundational aspect but by the direct application of formal methods to ordinary mathematical problems. In particular, the notion of first order (complete) theories provides a significant mathematical tool. And identifying specific virtuous properties of first order theories is a key part of that tool. In Subsection 4.1, we discuss the distinction (made for example by Bourbaki [24]) between the axiomatic method and formal methods. We argue a) that the study of formal theories is an effective mathematical tool for studying problems in mainstream mathematics and $b$ ) that the study of classes of theories (in particular, but not exclusively, by stability theoretic means) enhances this tool, identifies concepts that cross areas of mathematics and provides a useful scheme for organizing mathematics

Sections 4.1 and 4.2 contain basic arguments for a); these are further extended as b) is explicated in later sections. Subsection 4.2 argues that the notion of a complete theory selects classes of structures that are sufficiently similar that information can be transferred from one structure (where it may be verified using special properties of the structure) to another (which doesn't have those special properties). In Subsection 4.3, we move to claim b) by examining the role of categoricity. While categoricity is trivial for first order theories, categoricity in power is a notion with significant explanatory power. We describe the detailed information about the models of a theory $T$ that depend only on $T$ being categorical in power. Further, this analysis of categoricity in power leads to a general dimension theory for models of first order theories with broad applications to organize and to do mainstream mathematics. We sketch the stability hierarchy and its consequences in Subsection 4.4. Subsection 4.5 notes that the classification introduced in Subsection 4.4 sheds light on some traditional philosophical problems about infinity. In Subsection 4.6, we provide some examples of the uses of model theory in mathematics, stressing the connections to the hierarchy of properties of theories described in Subsection 4.4. Subsection 4.7 is a rudimentary example of the kind of case study that could be done to fill out our argument; we give a more extended treatment of groups of finite Morley rank. Subsection 5.1 provides a mathematical example (Lefchetz's principle) of the value of infinitary logic. We summarize the study of categoricity in $L_{\omega_{1} . \omega}$ in Subsection 5.2. In Subsection 5.3, we expound recent work of Hyttinen, Kangas, and Väänänen that invoke the first order analysis to obtain striking results on categoricity in infinitary second order logic. Section 6 summarizes the argument.

In addition to thanking Mic Detlefsen for providing and elucidating the original questions, I want to thank him as well as Andrew Arana, Juliette Kennedy, Saharon Shelah, Jouko Väänänen and the anonymous referee for many helpful discussions or comments.

§2. Framing the question. We address Question I along two axes, 'Why is the theory studied and what is the logic?'. But first we analyze the underlying issue. What are the criteria by which one property of a theory is judged better than another? We clarify some basic terminology in Subsection 2.1 and note 
the significance of apparently small changes in definitions. In Subsection 2.2, we provide our criterion for what makes a property of theories virtuous.

2.1. Terminology and its importance. The meanings of such words as categoricity and theory have varied over time and among different groups of logicians during the 20th century. Without giving a serious historical account I want to settle on particular meanings commonly taken by contemporary model theorists. Some of the subtle differences in these definitions are crucial for the uses of formalized theories described in Section 4.

In [15], I distinguished two degrees of formalization. The Euclid-Hilbert (the Hilbert of the Grundlagen) framework has the notions of axioms, definitions, proofs and, with Hilbert, models. But the arguments take place in natural language. For Euclid-Hilbert logic is a means of proof. In the Hilbert (the founder of proof theory)-Gödel-Tarski framework, logic is a mathematical subject. There are now explicit rules for defining a formal language and for proof. I gave the following anachronistic definition of a Hilbert-Gödel-Tarski ${ }^{3}$ formalization of an area of mathematics in [15].

Definition 2.1 (Formalization). A full formalization involves the following components.

1. Vocabulary ${ }^{4}$ : Specification of primitive notions.

2. Logic:

(a) Specify a class 5 of well formed formulas.

(b) Specify truth of a formula from this class in a structure $(M \models \phi)$.

(c) Specify the notion of a formal deduction for these sentences $(\Phi \vdash \phi)$.

3. Axioms: Specify the basic properties of the situation in question by sentences of the logic.

In [15], I focused on the development of a formal theory from prior intuitions of a mathematical subject area. In this paper, I contrast this development with the use of fully formalized theories as a tool in mathematics. In the latter context the particular axiomatization is irrelevant. Thus, I define: a theory $T$ is a collection of sentences in some logic $\mathcal{L}$, which is closed under semantic consequence ${ }^{6}$. (We will consider first order, second order, $L_{\omega_{1} . \omega}, L_{\kappa . \omega}^{2}$ and $L_{\omega_{1} . \omega}(Q)$.) For simplicity, we will assume that $T$ is consistent (has at least one model), has only infinite models, and is in a countable vocabulary. I assume the existence of a semantics for each logic is defined in ZFC.

\footnotetext{
${ }^{3}$ In some presentations I have extended the name to include Vaught. This is to emphasize that the modern notions of 'truth in a model' and 'elementary submodel' are firmly (perhaps for the first time?) established in [99].

${ }^{4}$ In Section 1 of [15] I elaborate on the special role attention to vocabulary plays in model theory.

${ }^{5}$ For most logics there are only a set of formulas, but some infinitary languages have a proper class of formulas.

${ }^{6}$ This is by no means a universal convention even in model theory. We adopt it to avoid a cumbersome use of the fairly trivial equivalence relation that theories, defined as sets of sentences, are equivalent if they have the same consequences. Even as late as Shoenfield's classic graduate text [94], the word theory is used for the entire syntactical apparatus: formal language, axioms and rules of inference for the logic, and specific axioms.
} 
$T$ is categorical if it has exactly one model (up to isomorphism).

$T$ is categorical in power $\kappa$ if it has exactly one model in cardinality $\kappa . T$ is totally categorical if it is categorical in every infinite power.

Definition 2.2. $\quad$ 1. $A \operatorname{logic}^{7} \quad \mathcal{L}$ is deductively complete if there is a deductive system such that for every $\bar{\phi}$

$$
\vdash \phi \text { if and only if } \models \phi \text {. }
$$

2. $A$ theory $T$ in a logic $\mathcal{L}$ is (semantically) complete if for every sentence $\phi \in \mathcal{L}$

$$
T \vDash \phi \text { or } T \models \neg \phi .
$$

3. $A$ theory $T$ in a logic $\mathcal{L}$ is (deductively) complete if for every sentence $\phi \in \mathcal{L}$

$$
T \vdash \phi \text { or } T \vdash \neg \phi \text {. }
$$

Under these definitions, every categorical theory is semantically complete. Further every theory in a logic which admits upward and downward Löwenheim-Skölem theorem for theories that is categorical in some infinite cardinality is semantically complete. First order logic is the only one of our logics that satisfies this condition without any qualification. Note that for any structure $M$ and any $\operatorname{logic} \mathcal{L}, \mathrm{Th}_{\mathcal{L}}(M)=\{\phi \in \mathcal{L}: M \vDash \phi\}$ is a complete theory.

A theory $T$ admits elimination of quantifiers if every formula $\phi(\mathbf{x})$ is equivalent in $T$ to a quantifier free formula $\phi^{*}(\mathbf{x})$. Elimination of quantifiers can arise in two radically different ways. Morley [71] introduced the idea of making a definitional extension of a first order theory by adding a predicate symbol for each definable relation. ${ }^{8}$ At the time this appeared only a technical convenience. In retrospect, it allows one to focus attention on the kind of property whose significance we explore in Subsection 4.4. Thus, most studies in pure model theory adopt the convention that this expansion has taken place. But this extension requires a large price; the vocabulary is no longer tied to the basic concepts of the area of mathematics. Thus algebraic model theorists work very hard to find the minimal number of extensions by definition ${ }^{9}$ that must be made to obtain quantifier elimination (or the weaker model completeness).

As we will see, apparently minor differences in terminology introduced only for convenience, such as demanding a theory is closed under semantic consequence, or completeness, or invoking Morley's procedure can signal major changes in viewpoint.

The model theoretic analyses discussed below depend on the description of some area of mathematics as the models of a formal first order theory. Actual formulas in the formal system appear rarely in the technical papers;

\footnotetext{
${ }^{7}$ While there is a standard deductive system for second order logic, whether second order logic is complete depends on the choice of semantics (Henkin or 'full').

${ }^{8}$ For more detail on the methodological role of this notion see Section 1.1 of [15].

${ }^{9} \mathrm{An}$ extension by definition or definitional extension of a first order theory is obtained by adding a new predicate symbol with an explicit definition. E.g.. in real closed fields (with symbols $(+, \cdot, 0,1)$, one adds the symbol < and the axiom $\forall x \forall y\left[x<y \leftrightarrow(\exists z) y-x=z^{2}\right]$.
} 
the fact that a particular concept admits such a description is endemic and essential.

2.2. A criterion for evaluating properties of theories. I leave the notion of a property of a theory undefined. But here are a number of examples of 'properties': categorical, complete, decidable, finitely axiomatizable, $\Pi_{2}^{0}$-axiomatizable, has an or only infinite models, interprets arithmetic. Another family of properties refer to models of the theory: each model admits $^{10}$ a linear order of an infinite subset; each model admits a combinatorial geometry; each model admits a tree-decomposition into countable models; each model interprets a classical group; every definable subset of a model is finite or cofinite; every model is linearly ordered and every definable subset is a finite union of intervals. The second group of properties (sometimes nontrivially) satisfies that for a complete theory, 'each model admits' is equivalent to 'some model admits'. Further examples of the second sort and the significance of that kind of property for mathematics will be developed below. Key to all of these notions is that they involve syntactic properties of the theory.

I take the word virtue in Question I to mean: the property of theories has significant mathematical consequences for any theory holding the property. For categoricity to be virtuous it would have to be that some properties of theories are explained simply by them being categorical. The notion of significant consequence is left vague now, but one aspect of significance is that theories which have the property will display other significant similarities. Many examples of 'significant consequence' appear in Section 4.4. We will note in Subsection 3 that the mere fact of categoricity has few consequences for a second order theory. In contrast, as we'll see in Subsection 4.3 the property, categoricity in power, of a first order theory $T$ gives rich structural information about the individual models of $T$.

A property could be virtuous because it has useful equivalents. For example, a first order theory $T$ is model complete if every submodel $N$ of a model $M$ is an elementary submodel, written $N \prec M$. This is equivalent to the statement: every formula $\psi(\mathbf{x})$ is proven equivalent in $T$ to a formula $(\exists \mathbf{y}) \phi(\mathbf{x}, \mathbf{y})$, where $\phi$ is quantifier free. The second version is of enormous help in analyzing the definable subsets of a model of $T$.

To determine the virtue of a property $A$ ask, 'What consequences for the theory $T$ or for models of the theory follow from $A$ holding of $T$ ?' We will be particularly interested in 'structural properties', i.e., information about how the models of the theory are constructed from simpler structures. In this context Shelah's notion of a dividing line lays out those properties which are 'most virtuous'.

A dividing line is not just a good property, it is one for which we have some things to say on both sides: the classes having the property and the ones failing it. In our context normally all the classes on one side, the "high" one, will be provably "chaotic" by the nonstructure side

\footnotetext{
${ }^{10}$ 'Admits' is jargon; it means the property described holds of or about the model.
} 
of our theory, and all the classes on the other side, the "low" one will have a positive theory. The class of models of true arithmetic is a prototypical example for a class in the "high" side and the class of algebraically closed fields, the prototypical nontrivial example in the "low" side."

We will argue in general that categoricity is not very virtuous. Its importance is as a signal that a theory (or rather an axiomatization) attempting to describe a particular structure has succeeded. But for first order theories, categoricity in power is a highly virtuous property of a theory ${ }^{12}$. Further we will argue that a small number of models in a cardinal is a sign that the models of the theory have a strong structure theory.

§3. Categoricity of second order theories. As we explain in Section 4.1, Löwenheim-Skolem conditions undermine the significance of categoricity in logics weaker than second order. In this section, we argue against categoricity per se as a significant property of second order theories, while acknowledging the importance of showing certain axiomatizations are categorical.

We need to distinguish here between the categoricity of an axiomatization $^{13}$ and the categoricity of a theory. One aim of axiomatization is to describe a particular, fundamental structure. There are really very few such structures. In addition to the reals $\Re$ and natural numbers $\mathbb{N}$ one could add $\mathbb{Z}, \mathbb{C}, \mathbb{Q}$ and of course Euclidean geometry. In the 20 th century such structures as the p-adic numbers enter the canon. Categoricity is a necessary condition for calling a second order axiomatization of a theory successful. But, given the ease described below of obtaining complete second order theories, it is not sufficient ${ }^{14}$. The goal of an axiomatization is to illuminate the central intuitions about the structure. The real linear order could be given a categorical axiomatization by describing the construction of the rationals from the natural numbers and then the reals as Cauchy sequences of rationals. As pointed out in [102], this construction takes place in $V_{\omega+7}$. But, it is Dedekind's categorical axiomatization of the real order as a (Dedekind) complete linear order that is mathematically useful. This axiomatization highlights the properties needed for the foundations of calculus [97]. From the perspective of providing a unique description of our intuitions, even a categorical second order axiomatization (of say the reals) is subject to attack from radically different perspectives (e.g., constructive mathematics or Ehrlich's absolute continuum [33]). Thus, the interest in categoricity is not

\footnotetext{
${ }^{11}$ See page 3 of [92]. Shelah elaborates this theme in Section 2. 'For the logically challenged' of the same chapter. Note that the virtue of a property in terms of the significance of its consequences is a different issue from which if either of 'high/chaotic' or 'low/structured' is virtuous under some conception of virtue.

${ }^{12}$ It does not however reach the status of dividing line; there are few specific consequences of a theory simply failing to be categorical in any power.

${ }^{13}$ Following modern model theoretic practice. I say a class is $\mathcal{L}$-axiomatizable if it is the class of models of a set of $\mathcal{L}$-sentences. If I mean recursively axiomatized I add this adjective.

${ }^{14}$ This perspective is highlighted by Huntington's initial name for categoricity, sufficiency, (See page 16 of [6]). Veblen [105] introduces the term categoricity.
} 
really that the theory ${ }^{15}$ is categorical but in the categoricity of the particular axiomatization that expresses the intuitions about the target structure.

For any logic $\mathcal{L}$ it is evident that categoricity of $\operatorname{Th}_{\mathcal{L}}(M)$ implies $\operatorname{Th}_{\mathcal{L}}(M)$ is semantically complete; the converse fails immediately if the logic has only a set of theories as there are a proper class of structures. Many second order theories are categorical. Consider the following little known results ${ }^{16}$.

Marek-Magidor/Ajtai $(\mathrm{V}=\mathrm{L})$ The second order theory of a countable structure is categorical.

H. Friedman $(\mathrm{V}=\mathrm{L})$ The second order theory of a Borel structure is categorical.

Solovay $(\mathrm{V}=\mathrm{L})$ A recursively axiomatizable complete second order theory is categorical.

Solovay/Ajtai It is consistent with $\mathrm{ZFC}$ that there is a complete finitely axiomatizable second order theory with a finite vocabulary ${ }^{17}$ that is not categorical.

To summarize these results, if a second order theory is complete and easily described (i.e., recursively axiomatized) or has an intended model which is 'small' (countable or Borel) then (at least ${ }^{18}$ in $L$ ) it is categorical. Awodey and Reck [6] point out that Carnap provided (as he realized) a false proof that every finitely axiomatized complete 2 nd order theory is categorical The Solovay/Ajtai result above shows this question cannot have a positive answer in ZFC. The fact that the most fundamental structures were categorical ${ }^{19}$ may partially explain why it took 25 years to clarify the distinction between syntactically complete and categorical. As reported in [6], Fraenkel in mid20s [36] distinguished these notions in a context of higher order logic without establishing that they are really distinct ${ }^{20}$.

\footnotetext{
${ }^{15}$ That is, the collection of all true sentences about the structure.

${ }^{16}$ These results appeared in a paper by Marek [63], its review by Magidor, and in a thread on Fom (http://cs .nyu . edu/pipermail/fom/2006-May/010544 .html). They were summarized by Ali Enayat at http://mathoverflow.net/questions/72635/categoricityin-second-order-logic/72659\#72659. Solovay's forcing argument for independence is at http://cs.nyu.edu/pipermail/fom/2006-May/010561.html.

Jouko Väänänen has relayed some further history. Corcoran reports in his review of [37], where Fraissé gives another proof of 1), that Fraissé had conjectured the first statement (about countable structures) in 1950. Further, Ajtai in [1] both proves that statement and that it requires $V=L$.

${ }^{17}$ There are trivial examples if infinitely many constants are allowed.

${ }^{18}$ In fact (per Väänänen), for countable models $V=L$ could be replaced by the existence of a second order definable well-ordering of the reals; in ongoing work Väänänen and Shelah are weakening this requirement.

${ }^{19}$ We follow current model theoretic practice and label a structure with any property that is satisfied by its complete first theory. We extend this practice by saying e.g., $M$ is 2 nd-order categorical when the 2 nd order theory of $M$ is categorical. Indeed the recent results cited above show that under $V=L$ each of the fundamental structures had to be categorical.

${ }^{20}$ More precisely Awodey and Reck point out that in the 2 nd edition of Fraenkel's book (1923) he had distinguished between categoricity and deductive completeness. In the 3rd (1928) edition he also clarifies the distinction between syntactic and semantic completeness. Corcoran [29] points out that Veblen in 1904 distinguishes deductive and semantic completeness but confuses semantic completeness and categoricity. Corcoran relates the generally
} 
I thank the referee for pointing me to a relevant line of research in this area, which involves categoricity in power for second order logic. Weaver and George $[108,109]$ make the following definitions. A Dedekind algebra is a structure for the vocabulary with one unary function. Each Dedekind algebra is determined by its configuration: the number of $\mathbb{Z}$-chains, the number of $\omega$-chains and for each $n$, the number of $n$-chains. The algebra $\mathcal{B}$ is finitely characterizable provided there is a finite subset of the second order theory of the algebra all of whose models are isomorphic. $\mathcal{B}$ is quasi-finitely characterizable provided there is a finite subset of the theory of the algebra all of whose models of the same cardinality are isomorphic. They then show that a Dedekind algebra is quasi-finitely characterizable if and and only if there is a second order sentence describing its configuration and categorical just when, in addition, the cardinal $|\mathcal{B}|$ is characterized by a second order mono-unary sentence.

This analysis largely reinforces the conclusions of the last paragraph. Those Dedekind algebras which have a fairly easily described structure are categorical in power. There is an important methodological distinction between this analysis and that in Theorem 4.9. The analysis of Dedekind algebras is restricted to a single vocabulary: one unary function. The first order analysis provides a structure theory for first order theories in any vocabulary, that are categorical in some uncountable power.

One might argue that it is hard to actually prove categority in ZFC. But for this argument to have much weight, one would have to get around two facts. 1) Consistently, ${ }^{21}$ categoricity is easy to achieve. Even in ZFC, there are many examples of categorical structures: various ordinals, the least inaccessible cardinal, the Hanf number of second order logic etc. 2) We have just seen that second order categoricity tells us nothing about the internal 'algebraic' properties of the structure. So the fact that a second order theory is categorical provides little information.

Bourbaki $^{22}$ wrote,

Many of the latter [mathematicians] have been unwilling for a long time to see in axiomatics anything other else than a futile logical hairsplitting not capable of fructifying any theory whatever. This critical attitude can probably be accounted for by a purely historical accident. The first axiomatic treatments and those which caused the greatest stir (those of arithmetic by Dedekind and Peano, those of Euclidean geometry by Hilbert) dealt with univalent theories, i.e., theories which are entirely determined by their complete systems of axioms; for this reason they could not be applied to any theory except the one from which they had been abstracted (quite contrary to what we have seen, for instance, for the theory of groups). If the same had been true

unsettled nature of these notions in the first decades of the 20th century and raises a number of precise historical questions.

${ }^{21}$ I.e., in some extension of ZFC.

${ }^{22}$ Page 230 of [24].Throughout the paper, square brackets in quotations indicate my interpolation; round brackets are from the original. 
of all other structures, the reproach of sterility brought against the axiomatic method, would have been fully justified.

Bourbaki has missed (ignored?) the fact that there are two different motivations for axiomatizing an area of mathematics. Bourbaki is focused on describing the properties of a class of structures that appear in many places in mathematics, specifically groups. Dedekind, Peano, and Hilbert provided second order axiomatizations that were explicitly intended to describe certain fundamental structures. Nevertheless from the Bourbaki standpoint of investigating the impact on mathematics as a whole of certain unifying concepts, one can reasonably ascribe sterility to such specific axiomatizations. However, the goal of the axiomatizers was to understand the given structure, not generalization. An insightful categorical axiomatization of a particular structure is expected only to explain the given structure, not to organize arguments about other structures. The other arguments mentioned above that provide many other categorical second order structures don't even have this benefit. Their real significance is to the understanding of second order logic, identifying some kinds of structures it can describe precisely (at least under $V=L)^{23}$. Even though (at least in $L$ ) there are many 2nd-order categorical structures, this fact tells us little about such structures. They have fairly simple descriptions, but not in a way that the reflects the properties of the structure. There is no consequence of the statement that the second order theory $T$ is categorical beyond 'it has only one model'. We will see the situation is far different for first order logic and categoricity in power.

§4. First order logic. In this section, we discuss many properties of first order theories which are virtuous in the sense of Subsection 2.2. Most were generated by Shelah's search for dividing lines in the spectrum problem ${ }^{24}$ of first order theories. Subsection 4.1 distinguishes between two uses of formalization. Subsection 4.2 addresses a second question of Detlefsen and shows the tight connection among the models of a fixed complete theory; thus complete theories become the natural class of theories to study. We introduce the notion of obtaining structural information about the models of a theory $T$ by global properties ${ }^{25}$ of $T$ with the motivating example of categoricity in power (Subsection 4.3). We introduce in Subsection 4.4 various formal (syntactic) properties that explain common properties of classes of complete first order theories and so are virtuous in the sense of Subsection 2.2. In addition, the classification imposed by these properties has a small finite

\footnotetext{
${ }^{23}$ The 'idea' of the arguments presented on Fom (See footnote 20) is that for well-ordered structures one can express in 2 nd order logic the assertion that a model is minimal (no initial segment is a model) provided that the axiomatization can be properly coded. The coding can be done in $L$. A similar approach (by Scott) proving that semantic completeness does imply categoricity for pure second order logic is Proposition 3 of [7].

${ }^{24} \mathrm{Calculate}$ the function $I_{T}(\kappa)$ that counts the number of nonisomorphic models of $T$ of cardinality $\kappa$.

${ }^{25}$ Here a global property of a theory such as categoricity or a place in the stability hierarchy is distinguished from local properties of the models of $T$.
} 
number of classes yielding a hierarchy of theories. Subsection 4.5 connects the stability hierarchy with classical problems of axiomatizing the infinite. Finally in Subsections 4.6 and 4.7, we explore how the properties described earlier in the section are exploited in current mathematical research.

4.1. The two roles of formal methods. While the immense significance of formal methods in the foundations of mathematics cannot be denied, the effect of such methods on the normal conduct of mathematics is open to considerable doubt $[60,80]$. Our goal here is not to join the current antifoundationalist parade but to describe a different contribution of formal methods to mathematics.

In [55] and [15], Juliette Kennedy and I have discussed the development of some 'formalism-free' approaches in logic and especially in model theory ${ }^{26}$. Here I proceed in the opposite direction; the goal is to show how formal symbolic logic plays an increasingly important role in ordinary mathematical investigations and provides schemes for organizing mathematics aimed not at finding foundations but at identifying mathematical concepts that link apparently diverse areas of mathematics and often address specific mathematical problems. We will see in Section 4.3 that these ideas develop from appropriate weakenings of categoricity and that they provided an unexpected fulfillment of some hopes of Bourbaki. We gave in Section 2.1 our definition of formalization of an area of mathematics [15]. What does this definition have to do with traditional mathematics? A leading representation theorist David Kazhdan, in the first chapter, entitled logic, of his lecture notes on motivic integration [54], writes:

One difficulty facing one who is trying to learn Model theory is disappearance of the natural distinction between the formalism and the substance. For example the fundamental existence theorem says that the syntactic analysis of a theory [the existence or nonexistence of a contradiction] is equivalent to the semantic analysis of a theory [the existence or nonexistence of a model].

At first glance this statement struck me as a bit strange. The fundamental point of model theory is the distinction between the syntactic and the semantic. On reflection ${ }^{27}$, it seems that Kazhdan is making a crucial point which I elaborate as follows. The separation of syntax and semantics is a relatively recent development. Dedekind grasps it late in the 19th century ${ }^{28}$. But it is actually clearly formulated as a tool only in Hilbert's 1917-18 lectures [96]. And immediately Hilbert smudges the line in one

\footnotetext{
${ }^{26}$ In Section 1.3 of that paper, I discussed Tarski's notion of a 'mathematical property' and won't elaborate further here.

${ }^{27}$ I thank Udi Hrushovski, Juliette Kennedy, and David Marker for illuminating correspondence on this issue.

${ }^{28}$ See footnote 11 of [95].
} 
direction by seeing the 'formal objects' as mathematical. As Sieg (page 75 of [95]) writes,

But it was only in his paper of 1904 , that Hilbert proposed a radically new, although still vague, approach to the consistency problem for mathematical theories. During the early 1920s he turned the issue into an elementary arithmetical problem...

Hilbert began the study of metamathematics by considering the formal language and the deduction relation on its sentences as mathematical objects. Thus, syntactical analysis is regarded as a study of mathematical objects

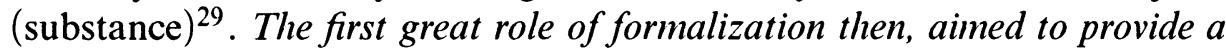
global foundation for mathematics. The Hilbert program treats the syntax as a mathematical 'substance'.

But Kazhdan is commenting on a smudge in the other direction; to prove the completeness theorem, Gödel constructs a model (a mathematical object) from the syntactical formulas ${ }^{30}$. When one views the completeness theorem solely from the standpoint of logic, the construction of 'models' from syntactic objects to make a statement about syntactic objects is less jarring. The surprise is when a real mathematical object arises from the syntactic paraphernalia. Marker e-mailed, 'I've found when lecturing that a similar stumbling block comes when giving the model theoretic proof of the Nullstellensatz (page 88 of [65]) or Hilbert's 17th Problem when the variables in the polynomial become the witnessing elements in a field extension.'

In [54], Kazhdan is not concerned with the global foundations of mathematics; he is concerned with laying a foundation for the study of motivic integration. This is an example of the second great application of formalization: By specifying in a formal language the primitive concepts involved in a particular area of mathematics and postulating the crucial insights of that field (usually thought of as defining the concepts implicitly in the Hilbert sense), one can employ the resources of 'formalization' to the analysis of 'standard' mathematical problems. The goal of this formalization is not foundational in the traditional sense but accords with Bourbaki's ideal of isolating the crucial constructions that appear in many places. The new feature is that one is able to identify certain common syntactic features in mathematical theories that discuss very different content. The bonus that Bourbaki did not foresee is the mathematical applicability of such resources as the completeness theorem, quantifier elimination, techniques of interpreting theories, and the entire apparatus of stability theory.

\footnotetext{
${ }^{29}$ This translation is seen even in the title of Post's 1920 thesis [77].

${ }^{30}$ In fact this blurring is frequent in the standpoint of the Schröder school of algebraic logic. Badesa makes this point in [9]; his argument is very clearly summarized in [5]. The combinatorics of the proofs of Löwenheim and Skolem are very close to those of Gödel. But Gödel makes the distinction between the syntactic and semantic clear since the warrant for the form of the syntactic configuration, which is interpreted as a model, is that it does not formally imply a contradiction.
} 
Bourbaki [24] distinguishes between 'logical formalism' and the 'axiomatic method'; the second, as they are too modest to say, is best exemplified by the Bourbaki treatise. ${ }^{31}$ 'We emphasize that it [logical formalism] is but one aspect of this [the axiomatic] method, indeed the least interesting one. ${ }^{32}$, In part, this remark is a reaction to the great pedantry of early 20th century logic as the language of mathematics was made rigorous. It also is a reaction to the use of logic only for foundational purposes in the precise sense of finding a universal grounding for mathematics. ${ }^{33}$ Bourbaki is reacting against a foundationalism which sacrifices meaning for verifiability. The coding of mathematics into set theory performs a useful function of providing a basis; unfortunately, the ideas are often lost in the translation. In contrast, the second role of formalization described above provides a means for analysis of ideas in different areas of mathematics.

In his remarks at the Vienna Gödel centenary symposium in 2006, Angus Macintyre wrote [60],

That the 1931 paper had a broad impact on popular culture is clear. In contrast, the impact on mathematics beyond mathematical logic has been so restricted that it is feasible to survey the areas of mathematics where ideas coming from Gödel have some relevance.

This sentence unintentionally makes a false identification. ${ }^{34}$ Macintyre's paper surveys the areas of mathematics where he sees the ideas coming from 'the 1931 paper' have some relevance. But incompleteness is not the only contribution of Gödel. In Section 4, we barely touch the tip of the iceberg of results across mathematics that develop from the Gödel completeness theorem and the use of formalization as we describe above. It is perhaps not surprising that in 1939, Dieudonné [32] sees only minimal value in formalization, 'le principal mérite de la méthode formaliste sera d'avoir dissipé les obscurités qui pesaieant encore sur la pensée mathématique'; the first true application of the compactness theorem in mathematics occurs only in Malcev's 1941 paper [62]. ${ }^{35}$

Bourbaki [24] hints at an 'architecture' of mathematics by describing three great 'types of structures': algebraic structures, order structures, and

\footnotetext{
${ }^{31}$ Mathias $[68,69]$ has earlier made a more detailed and more emphatic (stressing the inadequacy of their formal system) but similar critique to ours of Bourbaki's foundations. In particular, Mathias refers to Bourbaki's discussions with Rosser. William Howard [45] reports that Weil had the manuscript reviewed by Myhill and Tennenbaum (who were also at the University of Chicago) and rejected Myhill's negative comments.

${ }^{32}$ Parenthetical remarks added. Page 223 of [24].

${ }^{33}$ I use the term global foundations for this study.

${ }^{34}$ Macintyre has confirmed via email that he intended only to survey the influences of the incompleteness results.

${ }^{35}$ Malcev writes, 'The general approach to local theorems does not, of course, give the solutions to any difficult algebraic theorems. In many cases, however, it makes the algebraic proofs redundant.' Malcev goes on to point out that he significantly generalizes one earlier argument and gives a uniform proof for all cardinalities of an earlier result, whose proof by Baer held only for countable groups.
} 
topological structures. As we describe below in Subsection 4.4, the methods of stability theory provide a much more detailed and useful taxonomy which provides links between areas that were not addressed by the Bourbaki standpoint.

\subsection{Complete theories. Detlefsen asked.}

Question II (philosophical question) ${ }^{36}$ : Given that categoricity can rarely be achieved, are there alternative conditions that are more widely achievable and that give at least a substantial part of the benefit that categoricity would? Can completeness be shown to be such a condition? If so, can we give a relatively precise statement and demonstration of the part of the value of categoricity that it preserves?

We argue that the notion of a complete theory provides a formidable mathematical tool. One that is not sterile but allows for the comparison in a systematic way of structures that are closely related but not isomorphic. So we argue that completeness of a theory gives substantial benefits that approximate (especially in combination with further properties) the mathematical benefits of categoricity in power (but are completely impossible for categorical theories).

EXample 4.1 (Affine Schemes). Before turning to complete theories, we note that the notion of a theory provides a general method for studying 'families of mathematical structures'. First order axiomatizations may pick out salient features of a structure without determining the structure up to isomorphism. One obvious example is that algebraic geometers want to study 'the same' variety over different fields. This is crisply described as the solution in the field $k$ of the equations defining the variety. Similarly, the Chevalley groups can be seen as the matrix groups, given by a specific definition interpreted as solutions in each field. For finite fields, this gives 'most' of the nonexceptional finite simple groups [61]. These are examples of affine schemes. In introducing the notion of an affine group scheme, Waterhouse [107] begins with a page and a half of examples and defines a group functor. He then writes (chapter 1 of [107]),

The crucial additional ${ }^{37}$ property of our functors is that elements of $G(R)$ are given by finding solutions in $R$ of some family of equations with coefficients in $k$.... Affine group schemes are exactly the group functors constructed by solutions of equations. But such a definition would be technically awkward, since quite different collections of equations can have essentially the same solutions.

He follows with another page of proof and then a paragraph with a slightly imprecise (the source of the coefficients is not specified) definition of affine group scheme over a field $k$. Note that (for Waterhouse) a $k$-algebra is a commutative ring $R$ with unit that extends $k$. Now, for someone with a basic

\footnotetext{
${ }^{36}$ Question IV in Detlefsen's letter.

${ }^{37}$ My emphasis. See next paragraph.
} 
understanding of logic, an affine group scheme over $k$ is a collection of equations $\phi$ over $k$ that define a group under some binary operations defined by equations $\psi$. For any $k$-algebra $R$, the group functor $F$ sends $R$ to the subgroup defined in $R$ by those equations. ${ }^{38}$ The key point is that, not only is the formal version more perspicuous, it underlines the fundamental notion. Definability is not an 'addition'; the group functor aspect is a consequence of the equational definition.

We now give several examples that illustrate the mathematical power of the notion of complete theory, demonstrating that completeness is a virtuous property. As we said above, axiomatic theories arise from two distinct motivations. We discussed above the importance of a (usually second order) axiomatization of single significant structure such as $(\mathbb{N},+, \cdot)$ or $(\Re,+, \cdot)$. The other is to find the common characteristics of a number of structures; theories of the second sort include groups, rings, fields, etc. In the second case, little is gained simply from knowing a class is axiomatized by first order sentences. ${ }^{39}$ Although in Example 4.1, we exhibited a mathematical impact of formal axiomatizability, in general, the various completions of the theory simply provide too many alternatives. But for complete theories, the models are sufficiently similar that information can be transferred from one to another.

Kazhdan [54] (page 3) illuminates the key reason to study complete theories:

On the other hand, the Model theory is concentrated on [the] gap between an abstract definition and a concrete construction. Let $T$ be a complete theory. On the first glance one should not distinguish between different models of $\mathrm{T}$, since all the results which are true in one model of $\mathrm{T}$ are true in any other model. One of main observations of the Model theory says that our decision to ignore the existence of differences between models is too hasty. Different models of complete theories are of different flavors and support different intuitions. So an attack on a problem often starts [with] a choice of an appropriate model. Such an approach leads to many nontrivial techniques for constructions of models which all are based on the compactness theorem which is almost the same as the fundamental existence theorem.

On the other hand the novelty creates difficulties for an outsider who is trying to reformulate the concepts in familiar terms and to ignore the differences between models.

\footnotetext{
${ }^{38} \mathrm{We}$ are working with the incomplete theory $T$ in the language of rings with names for the elements of $k$. Two finite systems of equations over $k, \sigma(\mathbf{x})$ and $\tau(\mathbf{x})$, are equivalent if $T \vdash(\forall \mathbf{x}) \sigma(\mathbf{x})=\tau(\mathbf{x})$. The functorial aspects of $F$ are immediate from the preservation of positive formulas under homomorphism. Note that I am taking full advantage of $k$ being a field by being able to embed $k$ in each $k$-algebra. If we were to study modules over $\mathbb{Z}$ complications about finite characteristic would ensue. To be fair to Waterhouse's longer exposition he is introducing further terminology that is useful in his development.

${ }^{39}$ One does know such properties as closure of the class under ultraproduct, the ability to use compactness arguments, and, as illustrated above some consequences of the form of the axioms.
} 
The next three examples use the syntactic concept of a complete theory to transfer results between structures in way that was impossible or ad hoc without the formalism.

EXAMPLE 4.2 (Algebraic geometry). A model theoretic maxim asserts, 'algebraic geometry is the study of definable subsets of algebraically closed fields'. A first support for this assertion is Seidenberg's formulation [84] of the role of characteristic in the completeness of algebraically closed fields. The result follows from the completeness of algebraically closed fields of characteristic zero and Gödel's completeness theorem.

TheOREM 4.3 (Minor Principle of Lefschetz). Let $\phi$ be a sentence in the language $\mathcal{L}_{r}=\{0,1,+,-, \cdot\}$ for rings, where 0,1 are constants and,,$+- \cdot$ are binary functions. The following are equivalent:

1. $\phi$ is true in every algebraically closed field of characteristic 0 .

2. $\phi$ is true in some algebraically closed field of characteristic 0 .

3. $\phi$ is true in algebraically closed fields of characteristic $p$ for arbitrarily large primes $p$.

4. $\phi$ is true in algebraically closed fields of characteristic p for sufficiently large primes $p$.

In Subsection 5.1, we explore logicians' attempts to better reflect the Lefschetz principle using infinitary logic. But first we discuss algebraic geometry in the context of first order logic. Here are two equivalent statements of the same result showing the connections of formal definability ${ }^{40}$ to algebraic geometry.

THEOREM 4.4 (Chevalley-Tarski Theorem). ${ }^{41}$

Chevalley: Over an algebraically closed field, the projection of a constructible set is constructible.

Tarski: The theory of algebraically closed fields admits elimination of quantifiers.

Thus, quantifier elimination is a different phrasing of a fundamental tool of algebraic geometry. But there are too many definable sets; the basic domain of geometry is (unions) of varieties and this class is not closed under negation. This problem is addressed model theoretically by the notion of Zariski geometry ${ }^{42}$.

It frequently turns out that important information about a structure is only implicit in the structure but can be manifested by taking a saturated

\footnotetext{
${ }^{40} \mathrm{~A}$ relation on a field is 'constructible' if it is defined by a Boolean combination of equations. A set defined by a conjunction of equations is a variety.

${ }^{41}$ The version of this theorem for the reals is also known as the Tarski-Seidenberg theorem [83]. Tarski announced the quantifier eliminability of the real closed fields in [100]. He apparently became aware that his argument extended to the complex numbers when Robinson proved the quantifier eliminability of algebraically closed fields in [78]. There are rumors that Chevalley was well aware of Tarski's proof for the reals.

${ }^{42}$ Hrushovski and Zilber [49] provide a model theoretic context for the Zariski topology in which the solution sets of finite systems of equations are the closed sets.
} 
elementary extension of the theory. In particular, within the saturated models the syntactic types ${ }^{43}$ over a model can be realized as orbits of automorphisms of the ambient saturated model.

The notion of a generic point on a variety $X$ defined over a field $k$ is a rather amorphous concept for much of the twentieth century. In the model theoretic approach a generic point $a$ is a point in an extension field of $k$. More precisely, if $k$ is the algebraic closure of $k, a$ is a realization in an elementary extension of $\bar{k}$ of a nonforking extension of the type of minimal Morley rank and contained in $X$. Weil's notion of a universal domain is in model theoretic terms, an $\aleph_{1}$-saturated model of the theory of algebraically closed fields.

ExAmple 4.5 (Ax-Grothedieck Theorem). The Ax-Grothendieck theorem $[8,41]$ asserts that an injective polynomial map on an affine algebraic variety over $\mathbb{C}$ is surjective. The model theoretic proof ${ }^{44}$ in [8] observes that the condition is axiomatized by a family of 'for all -there exist' first order sentences $\phi_{i}$ (one for each pair of a map and a variety). Such sentences are preserved under direct limits and the $\phi_{i}$ are trivially true on all finite fields. So they hold for the algebraic closure of $F_{p}$ for each $p$ (as it is a direct limit of finite fields). By Theorem 4.3, $T=\operatorname{Th}(\mathbb{C})$, the theory of algebraically closed fields of characteristic 0 , is axiomatized by a schema $\Sigma$ asserting each polynomial has a root and, for each $p$, stating that the characteristic is not $p$. Since each $\phi_{i}$ is consistent with every finite subset of $\Sigma$, it is consistent with $\Sigma$ and so proved by $\Sigma$, since the consequences $T$ of $\Sigma$ form a complete theory.

Note that surjectivity implies bijectivity is false. A model theorist might immediately sense the failure since injectivity is universally axiomatized and so passes to substructure, while surjectivity is $\forall \exists$ axiomatized and so does not pass to substructures; an algebraist would immediately note that the map $x \mapsto x^{2}$ is a counterexample in, for example, the complex numbers.

Tao ([98]) gives an algebraic proof. He makes extensive use of the Nullstellensatz and notably misses the simpler direct limit argument to go from the

\footnotetext{
${ }^{43}$ Let $A$ be a subset of a model $M$ and $b \in M$. The syntactic type of $b$ over $A$ in the sense of $M$ is $\{\phi(x, a): a \in A, M \models \phi(b, a)\}$. A key reason for Shelah introducing the universal domain is to remove the dependence on the ambient $M$. A model $M$ is $\kappa$-saturated if every type over any $A$ with $|A|<\kappa$ is realized in $M$ and saturated if $\kappa=|M|$.

${ }^{44}$ There is a nonmodel theoretic proof in the spirit of $A x$ which replaces model completeness by multiple references to the Nullstellensatz [53]. Ax [8] was apparently unaware of Grothendieck's proof. He cites other work by Grothendieck and not this, but says "This fact seems to have been noticed only in special cases (e.g.. for affine space over the reals by Bialynicki-Birula and Rosenlicht [21])." While the argument in the real case is more complicated, it easily yields the result for the complexes.

In fact, there is no model theoretic proof of the real case. We can express the compactness argument in Ax's proof by noting $\mathbb{C}$ is an ultraproduct of locally finite fields. However, $\Re$ is not such an ultraproduct because $(\exists z)\left(x+z^{2}=y\right)$ defines a linear order of $\Re$. So this must be true for a family of fields indexed by a member of the ultrafilter if such an ultraproduct representation existed. But, no locally finite field can be linearly ordered.

Moreover, in this situation there are 'too many' definable sets. The natural variant for o-minimal theories that Nash functions (i.e., semialgebraic + real analytic functions) satisfy the theorem fails. $f(x)=\frac{x}{\left(x^{2}+1\right)^{\frac{1}{2}}}$ is a one-to-one map from $\Re$ into $(-1,1)$.
} 
finite fields to the algebraic closure of $F_{p}{ }^{45}$. These distinct approaches reflect the different perspective of logic on such a problem.

EXAmple 4.6 (Division Algebras). The study of finite dimensional division algebras provides a similar example.

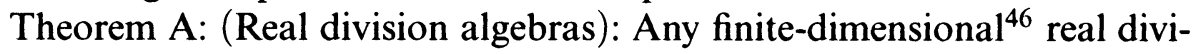
sion algebra must be of dimension $1,2,4$, or 8 . This is proved (by Hopf, Kervaire, Milnor 1958) using methods of algebraic topology (Bott periodicity $)^{47}$.

Theorem B: (Division algebras over real closed fields): Any finitedimensional division algebra over a real closed field must be of dimension $1,2,4$, or 8 .

Jacobson (page 314 of [52]) showed Theorem B follows from Theorem A by the completeness of the theory of real closed fields. No other proof is known.

Exactly what is involved in the topological proof? Does it formalize straightforwardly in the o-minimal topology on any real closed field? Or is there a reliance on the completeness of the reals?

This kind of direct transfer between models of complete (so necessarily formalized) theories is one benefit of formalization. Another is the ability to choose 'the right' complete first order theory. In a rough sense undecidability seems to disappear when a structure is 'completed' to answer natural questions (e.g. adding inverses and then roots to the natural numbers). Here is a specific measure of that idea.

EXAMPLE 4.7 (Ruler and Compass Geometry). Beeson [20] notes that the theory of 'constructible geometry' (i.e., the geometry of ruler and compass) is undecidable. This result is an application of Ziegler's proof [114] that any finitely axiomatizable theory in the vocabulary $(+, \cdot, 0,1)$ of which the real field is a model is undecidable. Thus, the complete theory is tractable while none of its finitely axiomatized subtheories are.

While I have given only a few simple examples, note that all the works in 'algebraic model theory' of A. Robinson, Ax-Kochen, Macintyre, Van den Dries and the entire school are using formal methods in the sense here described. Investigating the definable sets in models of a complete theory describing an appropriate area of mathematics becomes a powerful tool for studying that area.

4.3. Categoricity in power. In this subsection, we expound what we mean by 'significant mathematical consequences of a property' with a detailed

\footnotetext{
${ }^{45} \mathrm{He}$ gracefully acknowledges this simplification in reply to a comment.

${ }^{46}$ 'Real division algebra' appears in the literature with several meanings. For Frobenius they are associative and for Hurwitz multiplication by unit vectors is distance-preserving. Hopf, Kervaire, Milnor require only that the multiplication is continuous. Thus for $n=8$. the Dedekind completeness of the real numbers is used.

${ }^{47}$ Frobenius had shown the bound of 4 for associative division algebras in 1878 by a proof which extends immediately to all real closed fields.
} 
description of the consequences of categoricity in power. With this example in mind, in later sections, we give less detailed descriptions of the consequences of other properties.

Categoricity is trivial for first order logic. All and only finite structures are categorical in $L_{\omega . \omega}$. The interesting notion is 'Categoricity in (uncountable) Power'. The upward and downward Löwenheim-Skolem theorems show that for first order theories, categoricity in power of an axiomatization $\Phi$ implies the theory generated by $\Phi$ is complete. Ryll-Nardjewski [79], characterized first order theories that are $\aleph_{0}$-categorical. Unlike the second order case, this theorem contains a lot of information. We don't include all the technical definitions in the following description; they can be found in introductory texts in model theory (e.g. [65]).

THEOREM 4.8. The following are equivalent.

1. $T$ is $\aleph_{0}$-categorical.

2. $T$ has only finitely many finite n-types for each $n$.

3. $T$ has only finitely many inequivalent $n$-ary formulas for each $n$.

4. Thas a countable model that is both prime and saturated.

Wildly different kinds of theories are $\aleph_{0}$-categorical. The theory of an (infinite dimensional) vector space over a finite field differs enormously from the theory of an atomless Boolean algebra, the random graph or a dense linear order. But in the 1960s there was no way to make this difference precise. One distinction stands out; only the vector space is categorical in an uncountable power.

We discuss now the role of 'admitting a structure theory'. Theories that are categorical in an uncountable power have the simplest kind of structure theory and their study led to a more general analysis. In general, by a structure theory for an $\aleph_{1}$-categorical $T$ we mean the isolation of certain basic well-understood families of structures (e.g. geometries) usually identified by a single cardinal invariant, and the decomposition in a systematic way governed by these geometries of each model. The following theorem summarizes the basic landscape $[16,70,118]$ for first order theories categorical in (uncountable) power. Condition 4 gives the structural analysis of any $\aleph_{1}$-categorical theory.

THEOREM 4.9. (Morley/ Baldwin-Lachlan/Zilber) The following are equivalent:

1. $T$ is categorical in one uncountable cardinal.

2. $T$ is categorical in all uncountable cardinals.

3. $T$ is $\omega$-stable and has no two cardinal models.

4. Each model of $T$ is prime over a strongly minimal set.

5. Each model of $T$ can be decomposed by finite 'ladders' of strongly minimal sets $^{48}$.

\footnotetext{
${ }^{48}$ Zilber shows certain automorphism groups (the linking groups of the strongly minimal sets) are first order definable; this leads to the definability of the field in certain groups of finite Morley rank. See Subsection 4.7.
} 
First order theories which are totally categorical have a much stronger structure theory. Zilber's quest to prove that no totally categorical theory is finitely axiomatizable $[27,117]$ not only gave a detailed description of the models of such theories but sparked 'geometric stability theory'. Moreover, to eliminate the classification of the finite simple groups from the proof Zilber gave new proofs of the classification of two transitive groups $[35,115,116]$.

Because of Morley's theorem, for any theory we can say ' $\aleph_{1}$-categorical' for 'categorical in one (and therefore all) uncountable cardinals'. In addition [16] shows that an $\aleph_{1}$-categorical theory has either 1 or $\aleph_{0}$ models in $\aleph_{0}$. Since by 4 and 5 of Theorem 4.9, strongly minimal sets are the building blocks of uncountably categorical theories, we should describe them.

Definition 4.10. Let $T$ be a first order theory. $A$ definable subset $X$ of $a$ model of $T$ is strongly minimal if every definable subset $\phi(x, a)$ of $X$ is finite or cofinite (uniformly in a). $A$ theory $T$ is strongly minimal if the set defined by $x=x$ is strongly minimal in $T$.

The notion of a combinatorial geometry generalizes such examples as vector space closure or algebraic closure in fields. An important contribution of model theory is to find such geometries in many different contexts.

Definition 4.11. A pregeometry is a set $G$ together with a dependence relation

$$
c l: \mathcal{P}(G) \rightarrow \mathcal{P}(G)
$$

satisfying the following axioms.

A1. $\operatorname{cl}(X)=\bigcup\left\{\operatorname{cl}\left(X^{\prime}\right): X^{\prime} \subseteq\right.$ fin $\left.X\right\}$

A2. $X \subseteq \operatorname{cl}(X)$

A3. If $a \in \operatorname{cl}(X b)$ and $a \notin c l(X)$, then $b \in \operatorname{cl}(X a)$.

A4. $\operatorname{cl}(\operatorname{cl}(X))=\operatorname{cl}(X)$

If points are closed the structure is called a geometry.

Note that the preceding is a mathematical (formalism-free) concept. The next definition and theorem provide formal (syntactic conditions) on a theory for its models to be combinatorial geometries under an appropriate notion of closure.

Definition 4.12. Let $B \subset M \models T$ where $T$ is a complete theory. $a \in$ $\operatorname{acl}(B)$ (algebraic closure) if for some $\phi(a, y)$ and some $\boldsymbol{b} \in B, \phi(a, b)$ and $\phi(x, b)$ has only finitely many solutions. An acl-basis for a set $X$ is a maximal independent set with respect to this notion of closure.

THEOREM 4.13. A complete theory $T$ is strongly minimal if and only if it has infinite models, algebraic closure induces a combinatorial pregeometry on models of $T$ and, any bijection between acl-bases for models of $T$ extends to an isomorphism of the models.

The second condition is often rendered as, 'the pregeometry is homogeneous'; it is equivalent to say all independent sets of the same cardinality $\kappa$ realize the same type (in $\kappa$-variables). By Theorem 4.13 , the syntactic condition about the number of solutions of formulas leads to the existence of a geometry and a dimension for each model of the theory. 
The complex field or an infinite vector space over any field is strongly minimal. By Theorem 4.10 each model of a strongly minimal theory has a dimension ${ }^{49}$ generalizing that of vector spaces or the transcendence dimension in field theory. The dimension of a model of an arbitrary $\aleph_{1}$-categorical theory is the dimension of the strongly minimal set over which it is prime by Theorem 4.9.2. Thus a theory is $\aleph_{1}$-categorical if and only if each model is determined by a single dimension ${ }^{50}$. Sections 5 and 6 of Pillay's survey, Model Theory [74], gives an accessible and more detailed account of Zilber's [117] refinement, 'geometric stability theory', of Shelah's general classification program for first order theories. Approaching the Bourbaki ideal (Subsection 4.4), Zilber conjectured that all strongly minimal sets had a 'trivial' or 'vector-space like'(modular) or 'field-like' (nonmodular) geometry. Hrushovski found a counterexample [46] to Zilber's conjecture. His structure remains an outlier; but the method of construction led to many interesting developments. ${ }^{51}$ Hrushovski and Zilber [49] launched a program to rescue the conjecture (and better attune model theory to algebraic geometry). They analyzed the counterexamples that were at first sight pathologies by showing exactly how close 'ample Zariski structures' are to being algebraically closed fields. Zilber [121] lays out a detailed account of the further development of Zariski structures.

Moreover, this dimension theory extends to more general classes than the $\aleph_{1}$-categorical ones. For $\omega$-stable theories a dimension (Morley rank) can be defined on all definable subsets similar to and specializing to, in the case of algebraically closed field, the notion of dimension in algebraic geometry. While the notion of dimension is not a priori a syntactic notion, those appearing in model theory and algebraic geometry are.

If a theory is viewed as axiomatizing the properties of specific structure (e.g. the complex field) categoricity in power is the best approximation that first order logic can make to categoricity. But, it turns out to have far more profound implications than categoricity for studying the original structure. If the axioms are universal existential then the theory is model complete [59] (and under slightly more technical conditions admits elimination of quantifiers). Thus, the global property of categoricity in $\aleph_{1}$ determines the complexity of definable sets in the models. What can be very technical proofs of quantifier elimination by induction on quantifiers are replaced by more direct arguments for categoricity.

4.4. Virtuous properties as an organizing principle. There are two rather different approaches to choosing virtuous properties. One is to emphasize the simplicity of definitions in the theory; theories in which each formula is

\footnotetext{
${ }^{49}$ Dimension is a natural generalization of the notion of two and three dimensional space. The dimension tells us how many coordinates are needed to specify a point. This dimension (for a countable language) and uncountable strongly minimal (more generally $\aleph_{1}$-categorical) structure is the same as the cardinality of the model; [16] shows that for countable models either every model has dimension $\aleph_{0}$ or there are models of infinitely many finite dimensions.

${ }^{50}$ Strictly speaking, right to left requires $\omega$-stability.

${ }^{51}$ See $[12,14.106]$ for surveys.
} 
provably equivalent to one with no or at least few alternations of quantifiers are virtuous. However, for applications, as emphasized in the RobinsonMacintyre school, it is crucial that the primitive relations be understandable mathematical notions. In contrast, Morley introduced 'quantifier elimination by fiat'; extend the theory by definitions of the form $\phi(\mathbf{x}) \leftrightarrow R_{\phi}(\mathbf{x})$. This apparently technical shift lays the groundwork for Shelah's approach to finding a family of virtuous properties, which is our next topic.

The stability hierarchy is a collection of properties of theories as envisioned in Subsection 2.2 that organize complete first order theories (that is structures) into families with similar mathematically important properties. Bourbaki has some preliminary notions of combining the 'great motherstructures' (group, order, topology). They write, ${ }^{52}$ 'the organizing principle will be the concept of a hierarchy of structures, going from the simple to complex, from the general to the particular.' But this is a vague vision. We now sketch a realization of a more sophisticated organization of parts of mathematics.

Of course, one should realize that there have been sweeping and profound developments of the Bourbaki dream more directly in their school. Zalamea (page 140 [113]) summarizes, 'In Grothendieck's way of doing things, in particular, we can observe, firstly, the introduction of a web of incessant transfers, transcriptions, translations of concepts between apparently distant regions of mathematics, and, secondly, an equally incessant search for invariants, protoconcepts, and proto-objects behind that web of movements.' Grothendieck aims at the solution of specific problems that had arisen in the first half of the twentieth century. In contrast, Shelah looks for organization in the abstract; applications are fine but not essential. His response [93] on receiving the 2013 Steele prize makes clear his fundamental aims.

I am grateful for this great honour. While it is great to find full understanding of that for which we have considerable knowledge, I have been attracted to trying to find some order in the darkness, more specifically, finding meaningful dividing lines among general families of structures. This means that there are meaningful things to be said on both sides of the divide: characteristically, understanding the tame ones and giving evidence of being complicated for the chaotic ones. It is expected that this will eventually help in understanding even specific classes and even specific structures. Some others see this as the aim of model theory, not so for me. Still I expect and welcome such applications and interactions. It is a happy day for me that this line of thought has received such honourable recognition. Thank you.

While the exact meaning of Zalamea's 'transfers, transcriptions and translations' is unclear to me, the astonishing fact is that the properties isolated by Shelah without concern for applications show up in many areas of mathematics; we discuss some below. The most important of Shelah's innovations is to consider classes of theories defined by certain (syntactic) properties of

\footnotetext{
${ }^{52}$ Page 228 [24].
} 
the theory. Such classes include $\omega$-stable, stable, o-minimal, and 1-based. We first sketch Shelah's development of a collection of dividing lines which solved the test problem of calculating the spectrum ${ }^{53}$ of a first order theory. Then we will examine how this classification of theories has been developed and extended by many authors to obtain results across mathematics.

There are two key components to Shelah's solution of the spectrum problem:

1. Producing a sequence of dividing lines between chaos and structure such that

2. each model of a theory which satisfies 'the structural side' of each dichotomy is determined by a system of invariants.

The fundamental tool of this organization is the study of properties of definable relations, that is relations definable within a structure in a given formal language. Depending on the situation, there are several reasons why the subclass of definable sets is adequate to this task. In algebraic geometry (both real and complex) it turns out that mathematicians are, in fact, only studying (some) of the definable sets in the first place. In the other direction, the Wedderburn theory for noncommutative rings is on its face second order because of the study of ideals. But, for stable rings, there are enough first order definable ideals to obtain the classical structure theorems for stable rings $[10,17]$.

The general idea of a structure theory as in 2) above is to isolate 'definable' subsets of models of a theory that admit a dimension theory analogous to that in vector spaces. And then to show that all models are controlled by a family (indexed by a tree) of such dimensions. Thus, the notion of 'invariant' is vastly generalized to consider the different interaction of 'parts' of a structure that each have a dimension. Theories that are categorical in power are the simplest case. There is a single dimension and the control is very direct.

Shelah's stability theory [90] provides a method to categorize theories into two major classes (the main gap): those that admit a structure theory (classifiable) and those which are creative/chaotic. If a theory admits a structure theory, then all models of any cardinality are controlled by countable submodels via a mechanism which is the same for all such theories. In particular, this implies that the number of models in cardinality $\aleph_{\alpha}$ is bounded by $\beth_{\beta}(\alpha)$ (where $\beta<|T|^{+}$). In contrast, the number of models in $\aleph_{\alpha}$ of a chaotic theory is $2^{\aleph_{\alpha}}$; rather than adding another countable constituent, an essentially new method of creating models is needed in each cardinality.

In the last 25 years, tools in the same spirit of definability (but considering different syntactical properties) allow the investigation of the definable subsets of creative theories; these include the study of simple, o-minimal and theories without the independence property. While counting the number of models in each cardinality is the test question for this program, the greatest

\footnotetext{
${ }^{53}$ Footnote 24.
} 
benefit of studying this question lies in the development of tools to provide invariants and give detailed descriptions of each model.

The crucial point is that the stability hierarchy is defined by syntactic conditions $^{54}$. For example, a formula $\phi(\mathbf{x}, \mathbf{y})$ has the order property in a model $M$ if there are $\boldsymbol{a}_{i}, \boldsymbol{b}_{i} \in M$ such that

$$
M \models \phi\left(\boldsymbol{a}_{i}, \boldsymbol{b}_{j}\right) \text { iff } i<j .
$$

$T$ is stable if no formula has the order property in any model of $T$. But existentially quantifying out the $\boldsymbol{a}_{i}, \boldsymbol{b}_{i}, \phi$ is unstable in $T$ just if for every $n$ the sentence $\exists x_{1}, \ldots x_{n} \exists y_{1}, \ldots y_{n} \bigwedge_{i<j} \phi\left(x_{i}, y_{j}\right) \wedge \bigwedge_{j \geq i} \neg \phi\left(x_{i}, y_{j}\right)$ is in $T$. This last is clearly a syntactic condition. The (local) dimension theory of a stable theory which leads to the structural results follows from this syntactic condition.

A hidden existential quantifier in this definition disguises some of the significance. A theory $T$ is unstable if there is a formula with the order property. This formula may change from theory to theory. In a dense linear order one such is $x<y$; in a real closed field one is $(\exists z)\left(x+z^{2}=\right.$ $y)$; in the theory of $(\mathbb{Z},+, 0, \times)$ one is $\left(\exists z_{1}, z_{2}, z_{3}, z_{4}\right)\left(x+\left(z_{1}^{2}+z_{2}^{2}+\right.\right.$ $\left.z_{3}^{2}+z_{4}^{2}\right)=y$ ). In the theory of $(\mathbb{C},+, \times, \exp )$, one first notices that $\exp (u)=0$ defines a substructure which is isomorphic to $(\mathbb{Z},+, 0, \times)$ and uses the formula from its theory. It is this flexibility, grounded in the formal language, which underlies the wide applicability of stability theory. In infinite boolean algebras an unstable formula is $x \neq y \&(x \wedge y)=x$; here the domain of the linear order is not definable.

The Stability Hierarchy: Every complete first order theory falls into one of the following 4 classes.

1. $\omega$-stable

2. superstable but not $\omega$-stable

3. stable but not superstable

4. unstable

The stability hierarchy is essentially orthogonal to decidability. There are continuum many strongly minimal theories so most are not decidable. The random graph has the independence property but is recursively axiomatized and $\aleph_{0}$-categorical, so decidable. Similarly, the theory of atomless Boolean algebras has both the strict order property and the independence property but is decidable.

\footnotetext{
${ }^{54}$ In his classic 1961 paper [104], Vaught writes, 'One is tempted to say, by analogy with the discussion in the last paragraph of > 3 (where he asserts that finitely many $n$-types for each $n$ is syntactic), that condition 4.7.1 (countably many $n$-types for each $n$ ) is purely syntactical. Indeed, in 4.7.1, no reference to any semantical concept, such as "model". is made. However, a little thought convinces one that a notion of "purely syntactical condition" wide enough to include (.1) would be so broad as to be pointless.' Obviously, I am rejecting that observation. This is based partly on the experience that compactness arguments can be done around such requirements and partly on the observation that 'arithmetic' for the condition Vaught refers to and $\Pi_{1}^{1}$ for $\omega$-stability provide sufficiently constructive notions to justify 'syntactic'. It is, however, technically crucial for Shelah's development that the order property concerns a single formula, thus strengthening the applicability of compactness.
} 
The proof of the main gap relies on discovering several more dividing lines. We omit the technical definitions of the dimensional order property $(D O P)$, the omitting types order property (OTOP) and the shallow/deep dichotomy (discussed further in Subsection 5.3). Such properties ${ }^{55}$ lead to the existence of $2^{\kappa}$ (the maximum possible) nonisomorphic models of cardinality $\kappa$. If none of these properties hold the number of models is bounded well below $2^{\kappa}$ and there is good decomposition theorem for the models. Hart, Hrushovski, and Laskowski produced a full account of the spectrum problem for countable theories, including the greater intricacy for small infinite cardinalities in [43].

A common reading of Shelah is that the further down the above list a theory falls the more it lies on the side of chaos: 'chaos' means 'many models'. But Shelah has pointed out ${ }^{56}$ that this reading puts the cart before the horse, 'The aim is classification, finding dividing lines and their consequences. This should come with test problems. The number of models is an excellent test problem and few models is the strongest form of nonchaos.' But Shelah has suggested, beginning in late 70s, test problems for the study of theories with many nonisomorphic models, in particular, of unstable theories without the strict order property: existence of saturated extensions [87], the Keisler order (Chapter 6 of [86]) and the existence of universal models [57]. In [91] he extends this investigation by introducing the $S O P_{n}$ and establishing $\mathrm{SOP}_{4}$ as a dividing line for some purposes.

The study of o-minimality, simple theories and recent advances in studying nip (not the independence property ${ }^{57}$ )show that 'tame' is a broader category than stable. Among the canonical structures, the complex field is a prototype for good behavior and the real field (and even the real exponential field) are o-minimal and so there is sophisticated analysis of the definable sets. Of the canonical structures only arithmetic has so far resisted these methods of understanding. This is witnessed by its having both the independence property and being linearly ordered. Of course, set theory is equally unruly; a pairing function ${ }^{58}$ is incompatible with a global dimension theory.

In the last few paragraphs we have glimpsed the ways in which complete formal theories provide a framework for analysis. In Subsection 4.6, we discuss some of the profound implications of the hierarchy of complete theories for work in conventional mathematics. Thus, I argue that the study of complete theories a) focuses attention on the fundamental concepts of specific mathematical disciplines and $b$ ) even provides techniques for solving problems in these disciplines.

\footnotetext{
${ }^{55}$ The nonstructure arguments are not tightly linked to first order logic. They correspond to a theorem scheme for building many nonisomorphic models as Skolem hulls of sets of (linearly ordered or tree ordered) indiscernibles. The Skolem hull can accomodate some infinitary languages.

${ }^{56}$ See page 5 of [92].

${ }^{57}$ also called dependent

${ }^{58}$ A pairing function is a definable function from $X \times X$ to $X$. Such a function destroys a notion of dimension since each $n$-tuple is coded by a singleton.
} 
4.5. Axioms of infinity and the stability hierarchy. In this brief excursion, we discuss how the more advanced techniques of stability theory address a basic philosophical problem. How can one describe (prescribe) infinity? Here are three first order sentences which imply that the universe is infinite.

1. $<$ is a dense linear order.

2. $f(x)$ is an injective function; exactly one element does not have a predecessor.

3. $t(x, y)$ is a pairing function from $M \times M$ onto $M$.

W.W. Tait pointed out to me that these reflect three basic intuitions about how infinite sets arise: density, successor, and the observation that a number is smaller than its square. ${ }^{59}$ Are there other such intuitions?

The first example is nearly a complete theory; it becomes a complete finitely axiomatized theory by deciding whether there are end points. The second sentence has many finite models ( $n$-cycles). To make it a complete sentence with only infinite models requires infinitely many axioms; the resulting theory is $\aleph_{1}$-categorical. In contrast, there is an extension of a sentence expressing pairing function to a complete theory that is strictly stable and none that is superstable. If one strengthens completeness to demand that the sentence be categorical the answer is known but represents two major works.

THEOREM 4.14 (Zilber, Cherlin-Harrington-Lachlan). No first order sentence with only infinite models is categorical in all infinite cardinalities.

But such theories are quasi-finitely axiomatizable by a single sentence plus an 'infinity scheme' and there is a detailed structure theory for both finite and infinite models [28]. But the total categoricity is essential.

THEOREM 4.15 (Peretyatkin). There is an $\aleph_{1}$-categorical first order sentence.

Peretyatkin [72] was motivated by trying to capture a tiling problem but his example really seems to capture 'pairing'. The following question remains open: Is there a finitely axiomatizable strongly minimal set?

4.6. Formal methods as a tool in mathematics. In this section we give a very brief description of the use of formal tools in problems arising in other areas of mathematics. We give a short precis of applications in 5 fields of mathematics. Examples 1, 2, and 4 involve properties directly in the stability hierarchy; examples 3 and 4 involve the property of o-minimality; property 5) suggests a new sort of property. In Section 4.7, we give a slightly more detailed case study of one such example in order to lay out in more detail the kind of philosophical issues that such case studies can address. Each of the examples here, and still others would benefit from a similar, more detailed analysis.

Here is a primitive example of how informal mathematical notions can translate into the formal tools of stability theory. In an echo of the Bourbaki

\footnotetext{
${ }^{59}$ See footnote 58 .
} 
assertion of the importance of groups, in the presence of a group the stability conditions translate to chain conditions on 'definable subgroups' ${ }^{60}$ In an $\omega$-stable (superstable) theory there is no descending chain of definable subgroups (with infinite index at each stage). This principle is now seen to apply to different algebraic structures and gives a unified explanation for finding various kinds of radicals. ${ }^{61}$ (See [13] for a very early account of this phenomenon and $[2,3,38]$ for recent updates.)

The serious connections of stability theory with algebra and geometry grow out of geometric stability theory. Near the end of Subsection 4.3, we mentioned Zilber's trichotomy conjecture for strongly minimal sets. Shelah's notion of regular type extends the possible domains of combinatorial geometries to infinite rank types. In [50], Hrushovski studies the interaction of realizations regular types to obtain clearly mathematical information. The notation $r^{\alpha}$ means the type of $\alpha$ independent realizations of $r$. Roughly speaking, two types are orthogonal if there is 'no interaction' between realizations of the two types and almost orthogonal if there is no obvious interaction. In contrast, dominance indicates interactions and bidominance means the types, are very tightly connected.

TheOREM 4.16 (Hrushovski). Let $T$ be a stable theory. Let $\tilde{p}$ and $\tilde{q}$ be nonorthogonal stationary, regular types and let $n$ be maximal such that $\tilde{p}^{n}$ is almost orthogonal to $\tilde{q}^{\omega}$.

Then there exist $p$ almost bidominant to $\tilde{p}$ and $q$ dominated by $\tilde{q}$ such that: $n=1 q$ is the generic type of a (type) definable group that has the regular action on the realizations for $p$.

$n=2 q$ is the generic type of a (type) definable algebraically closed field that acts on the realizations for $p$ as an affine line.

$n=3 q$ is the generic type of a (type) definable algebraically closed field that acts on the realizations for $p$ as a projective line.

$n \geq 4$ is impossible.

Note that the hypotheses are purely model theoretic. There is no assumption that a group or ring is even interpretable in the theory. But the conclusion gives precise kinds of group and field actions that are definable in the given structures. This is one of many results from pure stability theory that has found applications in the last twenty five years; we sketch several areas of application.

EXAmple 4.17. 1. Differential Algebra: Ritt in the 1930's and later Kolchin developed the study of differential algebra. Abraham Robinson introduced the notion of a differentially closed field in analogy with the algebraically closed fields. This concept was incorporated in Kolchin's opus [58] and is one of the tools of differential algebraists. Blum [22] showed the theory was $\omega$-stable so differential closures were unique up to isomorphism. But Shelah [85] showed they were not minimal. Blum's proof is a direct application of Shelah's theorem showing

\footnotetext{
${ }^{60}$ In rings these subgroups become ideals.

${ }^{61} \mathrm{~A}$ 'radical' a technical term used in the structure theory of rings.
} 
the uniqueness of prime models over sets for $\omega$-stable theories. With some actual investigation of differential equations, he shows they need not be minimal.

For the continued interplay between various families of (differential) groups and rings and stability theory see [39]. Marker [66] provides an overall summary of the interaction of model theory and differential algebra. From our viewpoint, the crucial data is the definability conditions are applied either directly or by analogy in several areas of mathematics; the definability provides a uniform pattern.

2. Arithmetic algebraic geometry: Hrushovski's proof of Mordell-Lang for function fields integrates Shelah's notions of orthogonality and $p$-regularity and such notions from geometric stability theory as onebased with tools of arithmetic algebraic geometry. Both the study of stable fields [67] and the model theory of differential algebra play a role. See the collection [25] for a proof with background in both model theory and algebraic geometry, Hrushovski [47] for an overview and connections to stability theory and [82] for a more recent account of work in this area.

3. Real algebraic geometry and o-minimality: An important property which does not lie in the stability hierarchy is o-minimality. This property is restricted to those theories for which a symbol $<$ defines a linear order of the universe of each model. A theory is o-minimal if in every model, every definable set is a finite union of intervals. Even though the definition concerns only subsets of the universe, the celldecomposition theorem provides a clear understanding of definable sets in all dimensions. The following description of the motivations for o-minimality are taken from Alex Wilkie's Seminaire Bourbaki of November 2007 [112].

The notion of an o-minimal expansion of the ordered field of real numbers was invented by Lou van den Dries as a framework for investigating the model theory of the real exponential function $\exp : \Re \mapsto \Re: x \mapsto e^{x}$, and thereby settle an old problem of Tarski. More on this later, but for the moment it is best motivated as being a candidate for Grothendieck's idea of Ştame topology as expounded in his Esquisse d'un Programme. It seems to me that such a candidate should satisfy (at least) the following criteria. (A) It should be a framework that is flexible enough to carry out many geometrical and topological constructions on real functions and on subsets of real euclidean spaces. (B) But at the same time it should have built in restrictions so that we are a priori guaranteed that pathological phenomena can never arise. In particular, there should be a meaningful notion of dimension for all sets under consideration and any that can be constructed from these by use of the operations allowed under (A). (C) One must be able to prove finiteness theorems that are uniform over fibred collections. 
None of the standard restrictions on functions that arise in elementary real analysis satisfy both $(\mathrm{A})$ and $(\mathrm{B})$. For example, there exists a continuous function $G:(0,1) \mapsto(0,1)^{2}$ which is surjective, thereby destroying any hope of a dimension theory for a framework that admits all continuous functions. [...]

Rather than enumerate analytic conditions on sets and functions sufficient to guarantee the criteria (A), (B) and (C) however, we shall give one succinct axiom, the o-minimality axiom, which implies them. Of-course, this is a rather open-ended (and currently flourishing) project because of the large number of questions that one can ask under $(\mathrm{C})$. One must also provide concrete examples of collections of sets and functions that satisfy the axiom and this too is an active area of research.

Pillay and Steinhorn recognized o-minimality as a generalization of strong minimality. Strong minimality characterizes the definable subsets (no matter how extensive the ambient vocabulary) as easily describable using only equality (finite or cofinite). A theory is o-minimal if every definable subset is easily described in terms of a linear order of the model. Wilkie [1 11 ] proved that the real exponential field is o-minimal. A number of examples of further o-minimal structures were discovered; many are expansions of the real field. [30,64]. These techniques solved a problem of Hardy [103]. Although o-minimality is explicitly defined in terms of formal definability, in real algebraic geometry as in algebraic geometry in general, the only relations considered are definable. This has led to enormous integration between real algebraic geometry and model theory. Mancosu and Hafner in [42] analyze the real algebraic geometer Brumfiel's objections to transfer simply by completeness (as discussed in Example 4.6); the insights available from o-minimality far extend the analysis of the early 70s. For example, Pillay showed [73]: Let $G$ be a group definable in an o-minimal structure $M$. Then, $G$ can be equipped with a definable manifold structure making $G$ a topological group. In particular, if $M$ is an expansion of the reals then $G$ can be given the structure of a Lie group. The connection of o-minimality with Grothendieck's notion of 'tame topology' is examined at length by the geometer Teissier ${ }^{62}$.

4. Motivic integration: In what Scanlon [81] calls the prehistory, Denef shows the rationality of Poincare series; the geometers' tool of desingularization is avoided by an induction on quantifier rank using $p$-adic cell decomposition. The development of motivic integration theory in the 21 st century is a joint project of model theorists and geometers (including Denef/Cluckers/Hrushovski/Kazhdan/Loeser); it connects with the ideas of cell decomposition arising in the study of o-minimality and with issues arising from the study of $\mathrm{p}$-adically closed fields as NIP theories [44].

\footnotetext{
${ }^{62}$ See pages $232-236$ of [101].
} 
5. Asymptotic classes and finite groups: Macpherson and Steinhorn [61] define an asymptotic class as a class of finite models in which the number of solutions of a formula $\phi(\mathbf{x}, \boldsymbol{a})$ in a finite model $M$ can be uniformly approximated as $\mu M^{d / N}$ where $N$ is a parameter of the class and $\mu, d$ are uniformly defined depending on $\boldsymbol{a}$. This generalizes classical results on finding the number of solutions of diophantine equations in finite fields. But it also provides a scheme to try to explain the families of finite simple groups in terms of their definability.

4.7. Groups of finite Morley rank. In this subsection, we sketch how the resources of formalization, in particular, interpretation and the stability apparatus contribute to the study of a class of groups that were in fact defined by model theorists. This generalization of the study of finite simple groups illustrates the ways that formalization interacts with traditional mathematics and exhibits the power of formalization to introduce a generalizing principle. In this case the formalization (considering groups of finite Morley rank) provides a framework which includes both finite groups and algebraic groups over algebraically closed fields, thereby illuminating the role of finiteness conditions in each case. A group of finite Morley rank (FMR) is an infinite structure which admits a group operation and is $\omega$-stable with finite rank ${ }^{63}$ ; these notions require the formalism of the first order theory of the group in question. The driving conjecture:

CONJECTURE 4.18 (Cherlin/Zilber). A simple group of finite Morley rank is algebraic over an algebraically closed field.

links a model theoretic concept with algebraic geometry. The 25 year project to solve the conjecture has developed as an amalgam of basic stability theoretic tools with many different tools from finite and, recently, combinatorial group theory.

The basic scheme for understanding of the structure of groups relies on the Jordan-Hölder theorem: Each finite group can be written (uniquely up to the order of the decomposition) as $G=G_{0}>G_{1} \ldots G_{n}=1$, where $G_{i+1}$ is normal in $G_{i}$ and the quotient groups $G_{i} / G_{i+1}$ are simple. Thus, identifying the finite simple groups is a key step to understanding all finite groups. (We also need to know the nature of the extension at each level.) The analysis of FMR groups replaces induction on the cardinality of a group by induction on Morley rank.

Macintyre proved in the early 70 s that an $\aleph_{1}$-categorical, indeed any $\omega$-stable field is algebraically closed. An algebraic group is a variety $G$ over a field $k$ equipped with a group operation from $G \times G \rightarrow G$ that is a morphism (in the sense of algebraic geometry. ${ }^{64}$ The definition of an algebraic group (over an algebraically closed field) yields immediately that

\footnotetext{
${ }^{63}$ I am not giving a detailed historical survey here so many attributions and references are omitted. Poizat $[75,76]$ provides the general setting as in the late 1980s. Borovik and Nesin [23] gives a good overview of the finite rank case in the mid 1990s. Cherlin's webpage [26] lays out the Borovik program in broad strokes with references. A recent summary is [4].

${ }^{64} \mathrm{~A}$ by-product of the study under discussion is that an equivalent definition is: a group defined in an algebraically closed field.
} 
it is interpretable ${ }^{65}$ in an algebraically closed field and so has finite Morley rank. The algebraic definition of the dimension of an (in fact definable) subset yields the same value as the Morley rank.

Work in the late 1970s showed similar properties of algebraic groups over algebraically closed fields and groups of finite Morley rank in low rank. Cherlin extended from algebraic groups to FMR groups the propositions: rank 1 implies abelian; rank 2 implies solvable. Zilber showed that a solvable connected (see below) FMR group which is not nilpotent interprets an algebraically closed field. From this it ensues that every FMR group 'involves' an algebraically closed field; the issue is, 'how close is the involvement?'. Although algebraic groups over algebraically closed fields have FMR, groups of finite Morley rank are clearly more general. The Prüfer group $\mathbb{Z}_{\infty}$ is $\omega$-stable of rank 1 but not algebraic and FMR groups are closed under direct sum while algebraic groups are not. But the role of rank/dimension in each of the cases and the identification of the field in the group led to the idea that groups of finite Morley rank were some kind of natural closure of the algebraic groups. In particular, it led to Conjecture 4.18, that the basic building blocks are the same.

The classification of the finite simple groups identifies most of them as falling into families of algebraic groups over finite fields, the Chevalley groups. As we discussed in Example 4.1, families such as the Chevalley groups are a natural notion in model theory. They are the solution of the same definition of a matrix group as the field changes. A further impetus for this study is an analogy between finite groups and FMR groups. In the proofs, the descending chain conditions on all subgroups for finite subgroups is replaced by the descending chain condition on definable subgroups. This allows an algebraic definition of 'connected' replacing the topological definition in the study of algebraic groups. Induction on the cardinality of the group is replaced by induction on its Morley rank. The use of definability now provides a common framework for the study of algebraic and finite groups $^{66}$. The quest for Conjecture 4.18 has led to intricate analysis of all three generations of the proof of the classification of finite simple groups. In particular, the main strategy of the proof is an induction on the 'minimal counterexample' and the possibilities for this counterexample are sorted analogously to the finite case.

The following (slightly shortened) passage from the Borovik-Nesin book (page 29 [23]) shows the deep ties between the formal notion of 'interpretation' and algebra.

The notion of interpretation in model theory corresponds to a number of familiar phenomena in algebra which are often considered distinct: coordinatization, structure theory, and constructions like

\footnotetext{
${ }^{65}$ We don't spell out here the definition of an interpretation of one formal theory in another; any of the general sources in model theory mentioned above do so.

${ }^{66}$ Borovik had independently introduced the notion of ranked group (one which admits a collection of subsets containing the finite set and closed under (Boolean operation, projection, quotient). Poizat showed the class of such groups is exactly the FMR groups.
} 
direct product and homomorphic image. For example, a Desarguesian projective plane is coordinatized by a division ring; Artinian semisimple rings are finite direct products of matrix rings over divisions rings; many theorems of finite group theory have as their conclusion that a certain abstract group belongs to a standard family of matrix groups over [...]. All of these examples have a common feature: certain structures of one kind are somehow encoded in terms of structures of another kind. All of these examples have a further feature which plays no role in algebra but which is crucial for us: in each case the encoded structures can be recovered from the encoding structures definably.

Another role of the formalization is seen in the ability to focus on the key idea of a proposition. The standard statement of the Borel Tits theorem takes half a page and gives a laundry list of the possible kinds of maps (albeit considering the fields of definition of the groups). Zilber (See Theorem 4.17 $[75,76]$.) gives the following elegant statement.

Theorem 4.19 (Borel-Tits a la Zilber/Poizat). Every pure group isomorphism between two simple algebraic groups over algebraically closed fields $K$ and $L$ respectively can be written as the composition of a map induced by a field isomorphism between $K$ and $L$ followed by a quasi-rational function over $L$.

The maps are constructed as compositions of definable and abstract maps. Both the explanation of the role of interpretation in [23] and the statement and proof of the Borel-Tits theorem illustrate the role of formalization in providing context and clarity to mathematical results. While the development of this particular project takes place in the context of $\omega$-stable theories, the role of classes further down the Shelah hierarchy appeared in Examples 4.17.2 and 4.17.4.

§5. Infinitary logic. We consider in Subsection 5.1, the role of infinitary logic in formalizing mainstream mathematics by reviewing attempts to formalize the Lefschetz principle. Recall that $L_{\omega_{1} . \omega}$ allows countable Boolean combinations of formulas but only finite strings of first order quantifiers and $L_{\infty . \omega}$ strengthens the logic by allowing conjunctions of any cardinality. This analysis relies on the categoricity in uncountable powers of the theory of algebraically closed fields of a fixed characteristic. In Subsection 5.2, we describe the role of categoricity in power for the logic $L_{\omega_{1} . \omega}$. In Subsection 5.3, we return to second order logic with a slight twist. We consider second order logic with infinite conjunctions of various lengths.

5.1. Why infinitary logic?. We discuss in this section an example of logicians setting up local foundations for a subject. Here the foundations are tested against a goal set by André Weil. The Lefschetz principle was long known informally by algebraic geometers and appeared in Lefschetz' Algebraic Geometry. Barwise and Eklof [19] describe the issues around formalizing the Lefschetz principle as follows.

What we call Lefschetz principle has been stated by Weil as follows ([110], p 306): "for a given value of the characteristic $p$, every result, 
involving only number of points and varieties, which has been proved for some choice of universal domain remains valid without restriction; there is but one algebraic geometry of characteristic $p$; not one algebraic geometry for each choice of universal domain." Weil says that a formal proof of this principle would require a 'formal metamathematical' characterization of the type of proposition' to which it applies; "this would have to depend upon the 'metamathematical' i.e., logical analysis of all our definitions.

Seidenberg [84] argues that Weil's formulation is weaker than Lefschetz intended. Seidenberg gives a formulation ( the minor principle Theorem 4.3) in terms of the infinite family of complete theories, algebraically closed fields of various characteristics, which encompasses Weil's version. But Seidenberg argues that this does not really reflect mathematical practice and conjectures that the Lefschetz principle really needs to be formulated in terms of what he call almost-elementary sentences, a fragment of $L_{\omega_{1} .(\omega)}$. Barwise and Eklof [19] address Weil's question head-on. "Thus, in contrast to previous mathematical formulations of the Lefschetz principle which arose from general logical considerations, [...] our starting point has been an analysis of the definitions of algebraic geometry." They extend Example 4.3 to a transfer principle in an infinitary version of finite type theory to encompass such notions as integers, affine and abstract varieties, polynomial ideals, and finitely generated extensions of the prime field.

In [34], Eklof builds on work of Feferman to construct a simpler logic than that in [19], a many-sorted language for $L_{\infty . \omega}$. This many-sorted approach is implicit in the Hrushovski setting [25], but Hrushovski remains in the first order context taking advantage of the tools of first order stability theory.

5.2. $L_{\omega_{1}, \omega}$. In this subsection, we survey the status of categoricity and categoricity in power for sentences of $L_{\omega_{1}, \omega}$. The main results are in $[88,89]$; we give a systematic development in [11].

Since there are $2^{\aleph_{0}}$ inequivalent sentences and $2^{2^{\aleph_{0}}}$ theories but a proper class of structures, some theories must fail to be categorical. In contrast to the first order case, there are countable structures that are categorical for $L_{\omega_{1}, \omega}$. By the downward Löwenheim-Skolem theorem, no sentence of $L_{\omega_{1}, \omega}$ can have a unique model which is uncountable. But the $L_{\omega_{1} . \omega}$-theory of the reals is categorical.

A countable structure is categorical iff it has no proper $L_{\omega_{1}, \omega}$-elementary submodel. For sentences in $L_{\omega_{1}, \omega}$, categoricity in power $\aleph_{1}$ implies the existence of a complete sentence satisfied by the model of cardinality $\aleph_{1}$. It is open whether this implication holds for $\aleph_{2}$-categoricity in $L_{\omega_{1} . \omega}$.

The best generalization of Morley's theorem to $L_{\omega_{1} . \omega}$ is due to Shelah $[88,89]$. Shelah shows that one can more profitably study this subject by focusing on classes of the form $E C(T$, Atomic $)$, the class of atomic ${ }^{67}$ models of complete countable first order theory.

\footnotetext{
${ }^{67} M$ is atomic if each finite sequence from $M$ realizes a complete type over the empty set.
} 
The class of models of a complete sentence $\phi$ of $L_{()_{1} . \omega}$ is in 1-1 correspondence with an $E C(T$, Atomic)-class (Chapter 6 of [11]). Making this translation is a key simplification. An $E C(T$, Atomic $)$-class is excellent if for every finite $n$ it is possible to find a unique amalgamation of $n$ independent countable models in the class.

THEOREM 5.1 (Shelah 1983). Assume only ZFC. If $\boldsymbol{K}$ is an excellent $E C(T$, Atomic $)$-class then if it is categorical in one uncountable cardinal, it is categorical in all uncountable cardinals.

THEOREM 5.2 (Shelah 1983). Assume ZFC $+2^{\aleph_{n}}<2^{\aleph_{n+1}}$ for finite $n$. If an $E C(T$, Atomic $)$-class $\boldsymbol{K}$ is categorical in $\aleph_{n}$, for all $n<\omega$, then it is excellent.

Thus, for $L_{\omega_{1} . \omega}$ the study of categoricity in power is in a relatively complete state; the outstanding question from a foundational standpoint is whether the very weak generalized continuum hypothesis (VWGCH: for all $n, 2^{\aleph_{n}}<$ $2^{\aleph_{n+1}}$ ) is actually needed. There are only a few papers aimed at finding an extension to the stability hierarchy in this framework $([18,40])$.

There are important mathematical structures, e.g. complex exponentiation, which exhibit the Gödel phenomena and so cannot be analyzed by stability techniques in first order logic. However, Zilber $[11,119,120]$ has conjectured a means for such an analysis in the logic $L_{\left(\omega_{1} . \omega\right.}(Q)$. There is a vast literature on this subject featuring authors such as Kirby, Bays, and Gavrilovich as well as Zilber.

5.3. Déjà vu: Categoricity in infinitary second order logic. We rehearse here some recent results of Hyttinen, Kangas, and Väänänen [51] and Keskinen [56] that identify in a systematic way a proper class of categorical structures $^{68}$. Consider the logic $L_{\kappa . \omega}^{2}$ which allows first and second order quantification and conjunctions of length $\kappa$. In this family of logics, as $\kappa$ varies there are a class of sentences so the cardinality argument for the existence of noncategorical structures fails. In fact, every structure of cardinality $\kappa$ is categorical in $L_{\kappa^{+} . \omega}^{2}$. The goal is to identify those structures of cardinality $\kappa$ that are categorical in $L_{\kappa . \omega}^{2}(\kappa$ not $\kappa+)$. Since this work draws on the first order stability hierarchy discussed in Section 4.4 , we begin with more detail on the main gap theorem.

Any standard text in stability theory shows that if $T$ is stable then via the notion of nonforking an independence relation can be defined on all models of $T$ which generalizes the independence notion in combinatorial geometries discussed in Subsection 4.3. In general, the closure relation fails to be a geometry because $\operatorname{cl}(\operatorname{cl}(X)) \neq \operatorname{cl}(X)$. But on the set of realizations of a so-called regular type it is. Thus in a model $M$ and for any regular type $p$ with domain in $M$, we can define the dimension of the realizations of $p$ in $M$.

If $T$ is not stable or even not superstable, $T$ has $2^{\kappa}$ models in every uncountable $\kappa([90])$. If either DOP or OTOP (see Subsection 4.4) holds $T$ has the maximal number of models in each uncountable cardinal. If the dimensional order property and the omitting types order property do not

\footnotetext{
${ }^{68}$ The authors use 'characterizable' for what we call 'categorical'. See also [56].
} 
hold (NDOP and NOTOP), each model $M$ of cardinality $\kappa$ can be decomposed as a tree of countable submodels indexed by some tree $I$. The root of this tree, $M_{0}$ is a prime ${ }^{69}$ model. For $s \in I$, each $M_{s}$ has a set of up to $\kappa$ extension extensions $M_{s i}$ which are independent over $M_{s} ; M$ is prime over $\bigcup_{s \in I} M_{s}$. The systematic representation of a model as prime over a tree of (independent) submodels is a fundamentally new mathematical notion. The theory is shallow if there is a uniform bound over all models on the rank of the tree; essentially deep means this tree is not well-founded. If a theory satisfies NDOP, NOTOP and is shallow the theory is called classifiable.

For a classifiable theory the number of models in $\aleph_{\alpha}$ is bounded by $\beth_{\beta}(\alpha)$ where $\beta<|T|^{+}$is a bound on the rank of the decomposing tree for all models of $T$ (independently of cardinality). [90] claims that each model of such a theory is characterized by a sentence in a certain 'dimension logic'. Unfortunately, there are technical difficulties in the definition of this logic. However, the new paper [51] shows how to find such a categorical sentence in $L_{\kappa^{+}, \omega}^{2}$. Thus they obtain:

THeOREM 5.3 (Hyttinen, Kangas, and Väänänen). Suppose that $\kappa$ is a regular cardinal such that $\kappa=\aleph_{\alpha}, \beth_{1}(|\alpha|+\omega) \leq \kappa$ and $2^{\lambda}<2^{\kappa}$ for all $\lambda<\kappa$. The countable complete theory $T$ is classifiable if and only if for every model $M$ of $T$ with $|M|=\kappa$, the $L_{\kappa, \omega}^{2}$ theory of $M$ is categorical.

The deduction from classifiability is a highly technical argument that the decomposition of the models (and the dimensions of the types involved) can be defined in $L_{\kappa^{+} . \omega}^{2}$. Conversely, if a theory is not classifiable (i.e., lies on the chaotic side of the main gap), it has $2^{\kappa}$ models in $\kappa$. The conditions on $\kappa$ imply there are only $2^{<\kappa}$ sentences in $L_{\kappa . \omega}^{2}$ and $2^{<\kappa}<2^{\kappa}$ so there must be a sentence which is not categorical in the logic $L_{\kappa .(\omega)}^{2}$.

So using the virtuous properties developed in first order logic, the authors are able to uniformly identify a large family of structures with cardinality $\kappa$ that are categorical in $L_{\kappa . \omega}^{2}$. But categoricity is used in Huntington's role ${ }^{70}$ of 'sufficiency'. It is again a test of an axiomatization. In contrast to the ad hoc search for the axioms of the fundamental structure, Shelah's structure theorems provide a strategy for obtaining the axiomatization. While the axiomatizations of the fundamental structures informed us about the principles underlying proofs in the underlying number theory and real analysis, the existence of these axiomatizations inform us about the structure of the models of the underlying classifiable first order theory.

§6. Conclusion. We have posited a criterion for evaluating the virtue of a property of theories (in some logic): whether the property has significant mathematical consequences. In particular, whether the theories or more importantly the models of the theories, which have this property, display other significant similarities.

We agree that from the foundational standpoint of clarifying our intuitions about a canonical object, categoricity of a second order axiomatization plays

\footnotetext{
${ }^{69}$ That is, under an appropriate notion of submodel. it can embedded in every model of $T$.

${ }^{70}$ Footnote 14.
} 
an important role. And this is a mathematical as well as philosophical role. E.g., Dedekind provides a framework for proving results about the real numbers.

But we have argued that according to our criterion, categoricity of a theory is not very interesting for second order logic and trivial for first order logic. However, for first order logic, categoricity in power is very significant because all such theories in any vocabulary possess a dimension theory similar to prototypical examples such as vector spaces. The stability hierarchy provides both a classification of first order theories which calibrates their ability to support nice structure theories and the details of such a structure theory. The key to the structure is the definition of local dimensions extending the basic phenomena in theories which are categorical in power.

One slogan for model theory is the 'study of definable sets of a structure'. We use the more general term formalization to encompass the definability of classes of models as well as definability within a particular model. A key lesson of the last half century is how much the first study influences the second. But both exemplify the use of formal methods. The reason most of the work takes place in first order logic, despite its lesser expressive power, is the pervasive use of the compactness theorem. Thus not only formalization but a particular property of the formalization plays a central role.

This analysis builds on the distinction between 'logical formalism' and 'axiomatization' made by Bourbaki. ${ }^{71}$ But we think Bourbaki has missed the significance of 'logical formalism' for mathematics and perhaps even reverses the relative importance of the two methods. In Section 4 and in particular Subsection 4.6, we gave a series of examples of the use of a formal language as a tool for proving mathematical results. Thus, this paper is a counterpoint to our [15] where we discussed certain 'formalism-free' developments in model theory. In this paper we stress one of the dominant themes of model theory: the role of formal language in understanding mathematical questions. More than the use of formalism in seeking global foundations for mathematics these applications have real effect in mathematics. While mathematicians often study classes of models such as groups, model theory adds several layers. 1) Studying classes of very similar (i.e., elementarily equivalent structures) structures can deepen the study of a particular structure. 2) The properties of classes of theories introduced in stability theory provide new links between theories in different content areas of mathematics that enable the transfer of results and methods.

Formal methods in model theory impact other areas of mathematics in several ways: 1) some areas do study formally definable sets; the link is immediate once the area is formalized; 2) in other cases, the definable sets are sufficiently rich that ostensibly nonformalizable or at least not first order-formalizable concepts can be studied by model theoretic means ${ }^{72}$ and 3) syntactic properties in the stability hierarchy provide connections

\footnotetext{
${ }^{71}$ See quotation in Subsection 4.1.

${ }^{72}$ Here are two such situations: the study of chain conditions in rings discussed about half way through Subsection 4.4 and arithmetic algebraic geometry, Example 4.17, where several different formal theories interact.
} 
across mathematics that are not evident without the logical perspective. I summarize with a quote ${ }^{73}$ from Ehud Hrushovski's address [48] at the International Conference of Mathematicians in 1998:

Instead of defining the abstract context for [stability] theory, I will present a number of its results in a number of special and hopefully more familiar, guises: compact complex manifolds, ordinary differential equations, difference equations, highly homogeneous finite structures. Each of these has features of its own and the transcription of results is not routine; they are nonetheless readily recognizable as instances of a single theory.

The focus on model theory in this essay just reflects my background; similar examples of concrete applications of formal methods can be found in other areas of logic: proof mining; set theoretic resolution of the Whitehead problem and issues in general topology: applications of descriptive set theory to group representations, combinatorics and analysis; use of computability hierarchies in computer science, in differential geometry and in studying randomness; etc.. But that is another story.

\section{REFERENCES}

[1] M. Ajta1. Isomorphism and higher order equivalence. Annals of Mathematical Logic. vol. 16 (1979). pp. 181-203.

[2] Ricakdo DE Aldama. Definable nilpotent and soluble envelopes in groups without the independence property. Mathematical Logic Quarterly. vol. 59 (2013).

[3] T. Altinel and P. Baginski. Definable envelopes of nilpotent subgroups of groups with chain conditions on centralizers. Proceedings of the American Mathematical Society. vol. 142 (2014). pp. 1497-1506.

[4] T. Altinel. A. Borovik. and G. Cherlin. Simple groups of finite Morley rank. American Mathematical Society Monographs Series. American Mathematical Society. Providence. RI. 2008.

[5] J. Avigad. Review of the birth of model theory: Löwenheim's theorem in the frame of the theory of relatives. The Mathematical Intelligencer. (2006). pp. 67-71.

[6] S. Awodey and E. REck. Completeness and categoricity, part I: Nineteenth-century axiomatics to twentieth-century metalogic. History and Philosophy of Logic. vol. 23 (2002). pp. 1-30.

[7] - Completeness and categoricity, part II: Twentieth-century metalogic to twenty-first century semantics. History and Philosophy of Logic. vol. 23 (2002). pp. 77-94.

[8] JamEs Ax. The elementary theory of finite fields. Annals of Mathematics. vol. 88 (1968). pp. 239-271.

[9] C. Badesa. The birth of model theory. Princeton University Press. Princeton. NJ. 2004.

[10] P. BAginsKI. Stable $\aleph_{0}$-categorical alternative rings. 2012. preprint.

[11] JoHn T. Baldwin. Categoricity. Number 51 in University Lecture Notes. American Mathematical Society. Providence. RI. 2009. www.math.uic.edu/ jbaldwin.

[12] - A field guide to Hrushovski constructions. Report: http://www.math.uic.edu/ $\sim$ jbaldwin/pub/hrutrav.pdf.

[13] - Stability theory and algebra. Journal of Symbolic Logic. vol. 44 (1979). pp. 599-608.

[14] - Notes on quasiminimality and excellence. this JoURNAL. vol. 10 (2004). pp. 334-367.

[15] - Formalization, primitive concepts, and purity. Review of Symbolic Logic. (2013). pp. 87-128. http://homepages .math.uic. edu/ jbaldwin/pub/purityandvocab10. pdf.

[16] J.T. BALdWIN and A.H. LACHLAN. On strongly minimal sets. Journal of Symbolic Logic. vol. 36 (1971). pp. 79-96.

[17] J.T. BALDWIN and B. ROSE. א ${ }_{0}$-categoricity and stahility of ring. Journal of Algebra. vol. 45 (1977). pp. $1-17$.

\footnotetext{
${ }^{73}$ Emphasis inserted.
} 
[18] J.T. Baldwin and S. Shelah. Stability spectrum for classes of atomic models. Journal of Mathematical Logic. vol. 12 (2012). preprint. http://www . math. uic . edu/ jbaldwin/pub/shnew22.

[19] P. BARWISE and J. EKLOF. Lefschetz's principle. Journal of Algebra. vol. 13 (1969). pp. 554-570.

[20] M. BEESON. Constructive geometry, proof theory and straight-edge and compass constructions. http://www.michaelbeeson.com/research/talks/ConstructiveGeometrySlides.pdf.

[21] A. Bialynicki-Birula and M. Rosenlicht. Injective morphisms of real algebraic varieties. Proceedings of the American Mathematical Society. vol. 13 (1962). pp. 200-203.

[22] L. BLum. Generalized algebraic structures: A model theoretical approach. PhD thesis. MIT. 1968.

[23] A. Borovik and A. Nesin. Groups of finite Morley rank. Oxford University Press. Oxford. UK. 1994.

[24] Nicholas Bourbaki. The architecture of mathematics. The American Mathematical Monthly. vol. 57 (1950). pp. 221-232.

[25] E. BousCaren. editor. Model theory and algehraic geometry : An introduction to E. Hrushovski's proof of the geometric Mordell-Lang conjecture. Springer-Verlag. Heidelberg. 1999.

[26] G.L. CherLin. Algehraicity conjecture. webpage Algebraicityconjecture. 2004.

[27] G.L. Cherlin. L. Harrington. and A.H. LaChLan. $\aleph_{0}$-categorical, $\aleph_{0}$-stable structures. Annals of Pure and Applied Logic. vol. 28 (1985). pp. 103-135.

[28] G.L. Cherlin and E. Hrushovski. Finite structures with few types. Annals of Mathematics Studies. Princeton University Press. Princeton. NJ. 2003.

[29] JoHn CORCORAN. Categoricity. History and philosophy of logic. vol. I (1980). pp. 187-207. http://dx.doi.org/10.1080/01445348008837010.

[30] L. VAN den Dries. Tame topology and O-minimal structures. London Mathematical Society Lecture Note Series. vol. 248. 1999.

[31] M. Dethefsen. Completeness and the ends of axiomatization. Interpreting Goedel (J. Kennedy. editor). Cambridge University Press. 2014.

[32] JEAN DiEUdonnté. Lés methodes axiomatique modernes et les fondements des mathématique. Revue Scientique. vol. 77 (1939). pp. 224-232.

[33] P. Eнrцich. The absolute arithmetic continnum and the unification of all numbers great and small. this Journal. vol. 18 (2012). pp. 1-45.

[34] P. EkLof. Lefischetz's principle and local functors. Proceedings of the American Mathematical Society. vol. 37 (1973). pp. 333-339.

[35] D. Evans. Homogeneous geometries. Proceedings of the London Mathematical Society. vol. 52 (1986). pp. 305-327.

[36] A. Fraenkel. Einleitung in die Mengenlehre. Springer. Berlin. 1928. 3rd. revised edition.

[37] R. Frä̈ssé. Deux relations dénombrables, logiquement équivalentes pour le second ordre, sont isomorphes (modulo un axiome de constructibilité. Mathematical logic and formal systems. Lecture Notes in Pure and Applied Mathematics. vol. 94. Dekker. New York. 1985. pp. 161-182.

[38] J. Freitag. Isogeny in supertable groups. 2012. preprint. http://arxiv.org/abs/1106.0695.

[39] - Isogeny in supertable groups. Archive for Mathematical Logic. to appear. preprint. http://arxiv.org/abs/1110.1766.

[40] R. Grossibrga and Bradd HaRT. The classification theory of excellent classes. The Journal of Symbolic Logic. vol. 54 (1989). pp. 1359-1381.

[41] A. Grothendicck. Elements de géometrie algébriques (rédigés avec la collaboration de J. Dieudonné), IV Etudes Locale des Schémas et des Morphismes de Schémas. Publications mathématiques de l'IHÉS. vol. 28 (1966). pp. 5-255.

[42] J. Hafner and P. Mancosu. Beyond unification. The Philosophy of Mathematical Practice (P. Mancosu. editor). Oxford University Press. 2008. pp. 151-178.

[43] B. HaRt. Hrushovski E. and C. Laskowski. The uncountable spectra of countable theories. Annals of Mathematics. vol. 152 (2000). pp. 207-257.

[44] D. Haskell. E. Hrushovski. and H.D. MaCPherson. Stable domination and independence in algebraically closed valued fields. Lecture Notes in Logic. Association for Symbolic Logic. 2007.

[45] W. Howard. Comments on the relations of Bourbaki and logicians. 2013. http://homepages. math.uic. edu/ jbaldwin/pub/howonbour.pdf.

[46] E. Hrushovski. A new strongly minimal set. Annals of Pure and Applied Logic. vol. 62 (1993). pp. 147-166.

[47] - Stability and its uses. Current developments in mathematics, 1996 (Cambridge, MA). International Press. Boston. MA. 1997. pp. 61-103.

[48] - Geometric model theory. Proceedings of the International Congress of Mathematicians Vol. I (Berlin, 1998). 1998. pp. 281-302.

[49] E. HRUSHoVSKI and B. ZILBER. Zariski geometries. Bulletin of the American Mathematical Society. vol. 28 (1993). pp. 315-324. 
[50] Ehud Hrushovski. Almost orthogonal regular types. Annals of Pure and Applied Logic. vol. 45 (1989). pp. 139-155.

[51] Tapani Hyttinen. Kaisa Kangas. and Jouko Vä̈̈nänen. On second order characterizability. Logic Journal of the IGPL. vol. 21 (2013). 767-787. arXiv: 1208. 5167. doi 10. 1093/jigpal/jzs047.

[52] N. JACOBSON. Lectures in Ahstract Algebra III. University Series in Higher Mathematics. Van Nostrand. Princeton. NJ. 1964.

[53] M.C. Kang. Injective morphisms of affine varieties. Proceedings of the American Mathematical Society. vol. 119 (1993). pp. 1-4.

[54] David KaZHDAN. Lecture notes in motivic integration: Logic. 2006. http://www. ma. huji . ac. il/ kazhdan/Notes/motivic/b.pdf.

[55] Juliette KenNedy. On formalism freeness. this Journal. vol. 19 (2013). pp. 351-393.

[56] L. Keskinen. Characterizing all models in infinite cardinalities. Annals of Pure and Applied Logic. vol. 164 (2008). pp. 230-250.

[57] M. KoJman and S. Shelah. Non-existence of universal orders in many cardinals. Journal of Symbolic Logic, vol. 875-891 (1992). pp. 261-294. paper KhSh409.

[58] E. Kolchin. Differential algehra \& algehraic groups. Academic Press. New York. 1973.

[59] P. Lindstrom. On model completeness. Theoria. vol. 30 (1964). pp. $183-196$.

[60] Angus J. Macintyre. The impact of Gödel's incompleteness theorems on mathematics. Kurt Gödel and the foundations of mathematics (Baaz et al.. editors). Cambridge University Press. Cambridge. UK. 2011. pp. 3-26.

[61] D. Macpherson and C. Steinhorn. Definability in classes of finite structures. Finite and algorithmic model theory (Javier Esparza. C. Michaux. and C. Steinhorn. editors). London Mathematical Society Lecture Note Series. vol. 379. Cambridge University Press. Cambridge. UK. 2011. pp. 140-176.

[62] A.I. MALCEV. A general method for obtaining local theorems in group theory. The metamathematics of algebraic systems, collected papers: 1936-1967 (B.F. Wells. editor). North-Holland Publication Co.. Amsterdam. 1971.pp. 1-14. 1941 Russian original in Uceny Zapiski Ivanov Ped. Inst.

[63] Wiкток Marek. Consistance d'une hypothèse de Fraïssè sur la définissabilité dans un langage du second ordre. Comptes Rendus de l'Académie des Sciences. Série A-B. vol. 276 (1973). pp. All47-All 50.

[64] D. MARKER. Review of: Tame topology and o-minimal structures, by Lou van den Dries. Bulletin of the American Mathematical Society. vol. 37 (2000). pp. 351-357.

[65] D. Marker. Model theory: An introduction. Springer-Verlag. New York. 2002.

[66] — The number of countable differentially closed fields. Notre Journal of Formal Logic. vol. 48 (2007). pp. 99-113.

[67] D. Marker. M. Messmer. and A. Pillay. Model theory of fields. Springer-Verlag. Berlin. 1996.

[68] A.R.D. Mathias. The ignorance of Bourhaki. Mathematical Intelligencer. vol. 14 (1992).pp. 4-13. also in: Physis Riv. Internaz. Storia Sci (N.S.) vol. 28 (1991). pp. 887-904.

[69] - Hilhert, Bourhaki, and the scorning of logic. 2012. preprint. https://www. dpmms . cam. ac.uk/ ardm/logbanf inalmk.pdf.

[70] M. MoRLEY. Categoricity in power. Transactions of the American Mathematical Society. vol. 114 (1965).pp. 514-538.

[71] - Omitting classes of elements. The theory of models (Addison. Henkin. and Tarski. editors). North-Holland. Amsterdam. 1965. pp. 265-273.

[72] M. G. Peretyatkin. Finitely axiomatizable theories. Springer-Verlag. New York. 1997.

[73] A. Pillay. On groups and fields definable in o-minimal structures. Journal of Pure and Applied Algebra. vol. 55 (1988). pp. 239-255.

[74] - Model theory. Logic and Philosophy today, Part 1: Journal of Indian Council of Philosophical Research. 28. 2010. http: //www1 . maths . leeds . ac . uk/ pillay/model theory . jicpr. revised.pdf: reprinted in Logic and Philosophy Today. Studies in Logic 29 (\& 30). College Publications. London. 2011.

[75] Bruno Poizat. Groupes Stables. Nur Al-mantiq Wal-ma'rifah. 82. Rue Racine 69100 Villeurbanne France. 1987.

[76] - Stable groups. American Mathematical Society. Providence. RI. 2001. translated from Groupes Stables.

[77] E. Post. Introduction to a general theory of elementary propositions. From Frege to Godel: A sourcebook in mathematical logic, 1879-1931 (J. Van Heijenoort. editor). Harvard University Press. Cambridge. MA. 1967. pp. 264-283.

[78] A. Rominson. On predicates in algebraically closed fields. The Journal of Symbolic Logic. vol. 19 (1954). pp. 103-114.

[79] C. Ryll-Nardzewski. On categoricity in power $\leq \aleph_{0}$. Bulletin L'Académie Polonaise des Science, Série des Sciences Mathématiques, Astronomiques et Physiques. vol. 7 (1959). pp. 545-548. 
[80] GCERAld E. SaCks. Remarks against foundational activity. Historia Mathematica. vol. 2 (1975). pp. 523-528.

[81] SCANLON. Motivic integration: An outsider's tutorial. Lecture Durham. England. 2009. http://math.berkeley.edu/ scanlon/papers/scanlon_durham_motivic_integration_ outsiders_tutorial.pdf.

[82] T. SCAnlon. Counting special points: Logic, Diophantine geometry, and transcendence theory. Bulletin of the American Mathematical Society. vol. 49 (2012). pp. 51-71.

[83] A. Seidenierg. A new decision method for elementary algebra. Annals of Mathematics. vol. 60 (1954). pp. 365-374.

[84]- Comments on Lefschetz's principle. American American Monthly. vol. 65 (1958). pp. 685-690.

[85] S. Shelah. Differentially closed fields. Israel Journal of Mathematics. vol. 16 (1973). pp. 314-328.

[86] Classification theory and the number of nonisomorphic models. North-Holland. Amsterdam. 1978.

[87] - Simple unstable theories. Annals of Mathematical Logic. vol. 19 (1980). pp. 177-203.

[88] - Classification theory for nonelementary classes. I. The number of uncountable models of $\psi \in L_{\omega_{1} \omega}$ part $A$. Israel Journal of Mathematics. vol. 46 (1983). no. 3. pp. 212-240. paper 87a.

[89] - Classification theory for nonelementary classes. I. The number of uncountable models of $\psi \in L_{\omega_{1} \omega}$ part B. Israel Journal of Mathematics. vol. 46 (1983). no. 3. pp. 241-271. paper 87b.

[90] Classification theory and the number of nomisomorphic models. second edition. NorthHolland. 1991.

[91] _ . Toward classifying unstable theories. Annals of Pure and Applied Logic. vol. 80 (1996). pp. 229-255. paper 500 .

[92] - Classification theory for abstract elementary classes. Studies in Logic. College Publications www.collegepublications.co.uk. 2009. Binds together papers 88r. 600. 705.734 with introduction E53.

[93] — Response to the award of the 2013 steele prize for seminal research. A.M.S. Prize Booklet.

2013. p. 50. http://www. ams.org/profession/prizebooklet-2013.pdf.

[94] Joseph ShOENFIELd. Mathematical logic. Addison-Wesley. Reading. MA. 1967.

[95] W. SicG. Mechanical procedures and mathematical experiences. Mathematics and Mind

(A. George. editor). Oxford University Press. Oxford. 1994. pp. 71-117.

[96] — Hilbert's programs: 1917-1922. this Journal. vol. 5 (1999). pp. 1-44.

[97] M. SpIvaK. Calculus. Publish or Perish Press. Houston. TX. 1980.

[98] T. TAO. Infinite fields, finite fields, and the Ax-Grothendieck theorem. http://terrytao. wordpress.com/2009/03/07/inf inite-fields-finite-fields-and-the-ax-grothendiecktheorem/.

[99] A. TARski and R.L. VaUght. Arithmetical extensions of relational systems. Compositio Mathematica. vol. 13 (1956). pp. 81-102.

[100] Alfred TARski. Sur les ensemble définissable de nombres réels I. Fundamenta Mathematica. vol. 17 (1931). pp. 210-239.

[101] B. TeIsSIER. Tame and stratified objects. Geometric Galois actions I: Around Grothendieck's Equisse d'un Programme (L. Schneps and Pierre Lochak. editors). Cambridge University Press. 1997. pp. 231-242.

[102] JoUKo VÄÄNÄNEN. Second order logic or set theory. this Journal. vol. 18 (2012). pp. 91-121.

[103] L. van den Dries. A. Macintyre. and D. Marker. Logarithmic-exponential power series. Journal of the London Mathematical Society. (1997). pp. 417-434.

[104] R.L. VAUGht. Denumerable models of complete theories. Infinitistic methods. Proceedings of the Symposium on Foundations of Mathematics. Warsaw. 1959. Państwowe Wydawnictwo Naukowe. Warsaw. 1961. pp. 303-321.

[105] Oswald Veblen. A system of axioms for geometry. Transactions of the American Mathematical Society. vol. 5 (1904). pp. 343-384. 1904.

[106] F. WAGNer. Relational structures and dimensions. Automorphisms of first order structures (Kaye Richard et. al.. editors). Oxford. Clarendon Press. 1994. pp 153-180.

[107] WiLliam Waterhouse. Affine group schemes. Springer-Verlag. New York. 1979.

[108] G. Weaver. The model theory of Dedekind algebras. preprint. http://www.bu.edu/wcp/ Papers/Logi/LogiWeav.htm.

[109] G. WEAVER and B. GeORGE. The Fraenkel-Carnap question for limited higher-order languages. Bulletin of the Section of Logic. vol. 39 (2010). http://www.filozof . uni.lodz.pl/bulletin/pdf/ 39_12_1.pdf.

[110] ANDRE WeIL. Foundations of algebraic geometry. American Mathematical Society. 1962. 1st edition 1946 
[111] A. WILKIE. Model completeness results for expansions of the real field by restricted Pfaffian functions and exponentiation. Journal of American Mathematical Society. (1996). pp. 1051-1094.

[112] . O-minimal structures. Séminaire BOURBAKI. 985. 2007. http: //eprints .ma.man . ac.uk/1745/01/covered/MIMS_ep2012_3.pdf.

[113] F. ZaLAMEA. Filosofia sintética de las matematáticas comtemporáneas. Colección OBRA SELECTA. Editorial Univeridad National de Colombia. Bogota. 2009.

[114] M. ZiEGLER. Einige unentscheidbare klorpertheorien. Enseignement Mathématique. vol. 28 (1982). pp. 269-280. Michael Beeson has an English translation.

[115] B.I. ZIL Ber. Strongly minimal countably categorical theories. Siberian Mathematics Journal. vol. 24 (1980). pp. 219-230.

[116] - Strongly minimal countably categorical theories II. Siherian Mathematics Journal. vol. 25 (1984). pp. 396-412.

[117] - The structure of models of uncountably categorical theories. Proceedings of the International Congress of Mathematicians August 16-23, 1983, Warszawa, pages 359-368. Polish Scientific Publishers. Warszawa. 1984. http://www .mathunion.org/ICM/ICM1983.1/Main/icm1983.1.0359. 0368. ocr.pdf.

[118] Uncountably categorical theories. Translations of the American Mathematical Society. American Mathematical Society. 1991. summary of earlier work.

[119] . Pseudo-exponentiation on algebraically closed fields of characteristic 0. Annals of Pure and Applied Logic. vol. 132 (2004). pp. 67-95.

[120] . A categoricity theorem for quasiminimal excellent classes. Logic and its applications, Contemporary Mathematics. American Mathematical Society. 2005. pp. 297-306.

[121] _ Zariski geometries: Geometry from the logicians point of view. Number 360 in London Math. Soc. Lecture Notes. London Mathematical Society. Cambridge University Press. 2010.

\author{
DEPARTMENT OF MATHEMATICS \\ STATISTICS AND COMPUTER SCIENCE M/C 249 \\ UNIVERSITY OF ILLINOIS AT CHICAGO \\ CHICAGO. IL 6067. USA
}

